\title{
Low-energy limit of the $O(4)$ quark-meson model from the functional renormalization group approach
}

\author{
Jürgen Eser, ${ }^{1, *}$ Florian Divotgey, ${ }^{1, \dagger}$ Mario Mitter, ${ }^{2, \$}$ and Dirk H. Rischke ${ }^{1,3, \S}$ \\ ${ }^{1}$ Institut für Theoretische Physik, Johann Wolfgang Goethe-Universität, Max-von-Laue-Straße 1, \\ D-60438 Frankfurt am Main, Germany \\ ${ }^{2}$ Department of Physics, Brookhaven National Laboratory, Upton, New York 11973, USA \\ ${ }^{3}$ Interdisciplinary Center for Theoretical Study and Department of Modern Physics, University of Science \\ and Technology of China, Hefei, Anhui 230026, China
}

(Received 6 April 2018; published 18 July 2018)

\begin{abstract}
We compute the low-energy limit of the $O(4)$-symmetric quark-meson model as an effective field theory for quantum chromodynamics (QCD) within the functional renormalization group (FRG) approach. In particular, we analyze the renormalization group flow of momentum-dependent pion self-interactions beyond the local potential approximation. The numerical results for these couplings obtained from the FRG are confronted with a recent tree-level study. Additionally, their effect on the wave-function renormalization factors and the curvature masses is investigated.
\end{abstract}

DOI: 10.1103/PhysRevD.98.014024

\section{INTRODUCTION}

The fundamental dynamics of the strong interaction is described by QCD. In the case of $N_{f}$ massless quark flavors, the classical Lagrangian of QCD possesses a global $S U\left(N_{f}\right)_{L} \times S U\left(N_{f}\right)_{R} \times U(1)_{L} \times U(1)_{R}$ flavor symmetry. Due to an anomaly [1], the $U(1)_{L} \times U(1)_{R}$ part of the symmetry is broken to $U(1)_{V} \equiv U(1)_{L+R}$, which corresponds to quark number conservation. We exclude this $U(1)_{V}$ symmetry from the following discussion, since it is trivially fulfilled in models with hadronic degrees of freedom (d.o.f.), like the quark-meson model. For this work, the relevant flavor symmetry is then given by $S U\left(N_{f}\right)_{L} \times S U\left(N_{f}\right)_{R}$, the so-called chiral symmetry, which is further broken both explicitly and spontaneously.

The experimentally observed hadrons can be grouped into the irreducible representations of $S U\left(N_{f}\right)_{V} \equiv S U\left(N_{f}\right)_{L+R}$, but not into those of $S U\left(N_{f}\right)_{L} \times S U\left(N_{f}\right)_{R}$. This signals the spontaneous breakdown of chiral symmetry to its diagonal flavor subgroup $S U\left(N_{f}\right)_{V}$. An immediate consequence of this symmetry-breaking mechanism is the occurrence of $N_{f}^{2}-1$ (pseudo-)Nambu-Goldstone bosons (pNGBs). The

\footnotetext{
*eser@th.physik.uni-frankfurt.de

fdivotgey@th.physik.uni-frankfurt.de

*mitter@bnl.gov

${ }^{\S}$ drischke@th.physik.uni-frankfurt.de
}

Published by the American Physical Society under the terms of the Creative Commons Attribution 4.0 International license. Further distribution of this work must maintain attribution to the author(s) and the published article's title, journal citation, and DOI. Funded by SCOAP. fact that these bosons are only very light instead of being massless indicates that the physical quarks have small finite masses, which break chiral symmetry explicitly. Throughout the rest of this work, we restrict ourselves to the case of two dynamical quark flavors, $N_{f}=2$, where the resulting three pNGBs are identified with the pion isotriplet $\vec{\pi}$.

An important property of QCD is that its coupling $\alpha_{S}$ becomes large at low energies. This implies that perturbation theory cannot be used to study the low-energy regime of this theory. Therefore, one has to use methods that do not rely on a perturbative expansion in powers of $\alpha_{S}$. One such possibility is given by effective field theories (EFTs). A crucial guiding principle in the construction of EFTs for QCD is chiral symmetry, which can either be realized in a linear [2-4] or a nonlinear way [5-7]. The latter way of realizing a symmetry results in an EFT describing the interaction of pNGB fields, i.e., of pions for $N_{f}=2$, among themselves. There, the pNGBs explicitly enter the theory as local coordinates parametrizing the vacuum manifold of the theory.

The most prominent EFT is given by chiral perturbation theory (ChPT) [8,9], whose connection to the low-energy regime of QCD has been studied in great detail in Ref. [10]. ChPT is defined by a Lagrangian that contains all chiral invariants that are obtained from a systematic expansion in powers of derivatives of the pion fields. The coupling constants that enter this expansion are usually referred to as low-energy constants (LECs). Because this expansion contains arbitrarily high powers of derivatives of the pion fields, the resulting Lagrangian is not perturbatively renormalizable. However, the aforementioned expansion can also be understood as a power series in $p /\left(4 \pi f_{\pi}\right)$, where $p$ 
denotes the momentum of the pion fields and $f_{\pi}$ the pion decay constant. This power series is expected to converge for small enough pion momenta, and all infinities can be absorbed order by order into the LECs.

As already mentioned before, it is also possible to realize chiral symmetry in a linear way. The resulting models are referred to as linear sigma models (LSMs) [11,12], which incorporate the pNGBs as well as their chiral partners on the same footing. Various versions of these models, also with vector and axial-vector mesons $[13,14]$, were subject to comprehensive studies over the last decades. The relation between ChPT and hadronic models based on a linear realization of chiral symmetry was studied in Refs. [15-17]. Concerning the low-energy limit, it was shown that the LECs of the most simple version of the LSM, including only the pion fields and the scalar sigma field $\sigma$, do not assume the same values as for QCD [8].

Recently, the so-called extended linear sigma model (eLSM) was developed. This model contains all groundstate quark-antiquark mesons with (pseudo)scalar and (axial-)vector quantum numbers up to $2 \mathrm{GeV}$ in mass. The Lagrangian of the eLSM respects all symmetries of QCD and also reflects their possible breaking patterns. The eLSM was studied for $N_{f}=2,3,4$ in Refs. [18-21]. Baryons were included as well and studied in vacuum [22-24] and at finite density $[25,26]$. From the requirement of dilatation symmetry and incorporating only positive semi-definite powers of the dilaton field, it follows that the eLSM Lagrangian contains only a finite number of terms. This results in a finite number of coupling constants and parameters, which have been determined in a global fit to experimentally measured masses and decay widths. It turns out that the eLSM is in remarkably good agreement with experimental data [20], such as meson masses and decay rates in the scalar-isoscalar sector, the $\eta-\eta^{\prime}$ mixing angle, as well as branching ratios for $a_{0}$.

The question whether the low-energy limit of the eLSM is consistent with that of QCD has been addressed in a recent tree-level study [27]. It turned out that the tree-level values of the LECs of the eLSM are in good overall agreement with the ChPT values. The obvious next step is then to extend this study in order to check whether loop contributions to the LECs are negligible or not. In this paper, we perform a first step in this direction by computing the loop corrections to the LECs of the $O(4)$ quark-meson model as a simplified version of the mesonic eLSM to which we add a Yukawa coupling of mesons to quark fields. In future work, we will extend this towards a study of loop corrections to the LECs of the full eLSM.

A framework that naturally generalizes the method presented in Ref. [27] is given by the functional renormalization group (FRG) [28], which has been employed to consider low-energy effective theories as well as QCD under various (thermodynamical) aspects; see, for instance, Refs. [29-49]. By applying the FRG technique in this work, we shed light on the low-energy limit of the $O(4)$ quark-meson model. More precisely, we compute loop corrections to the tree-level low-energy couplings of this model by means of including momentum-dependent meson vertices into the renormalization-group flow. In a first approximation (the validity of which will be checked), we will restrict ourselves to pion self-interactions. Our study can be viewed as an extension of the work [17] where, in the local potential approximation (LPA) including scale-dependent wave-function renormalization factors as well as a scale-dependent Yukawa coupling between mesons and quarks, it was shown that the FRG approach is able to produce the correct chiral logarithms in the expressions for the pion decay constant and the pion mass.

This paper is organized as follows: Sec. II introduces the concepts and methods that are used throughout this work. In Sec. II A, we briefly review the $O(4)$ LSM and summarize its tree-level low-energy limit. After recalling the basics of the FRG approach in Sec. II B, we derive the low-energy effective pion action for the $O(4)$ quark-meson model in Sec. II C. The corresponding FRG flow equations are presented in the Appendix. Finally, in Sec. III, we show a comparison of the FRG study and the tree-level estimate for the low-energy couplings. Our conclusions and an outlook for future investigations are given in Sec. IV.

\section{METHODS}

\section{A. $O(4)$ linear sigma model}

As mentioned in Sec. I, the eLSM is a hadronic model that comprises (pseudo)scalar and (axial-)vector mesons. Assignments of these fields to physical resonances can be found in Refs. [20,27].

In the $O(4)$ limit, the mesonic d.o.f. are described by the following matrix:

$$
\Phi=\sigma t_{0}+i \vec{\pi} \cdot \vec{t},
$$

where $t_{0}=\mathbb{1}_{2} / 2$ and $\vec{t}=\vec{\tau} / 2$. The vector $\vec{\tau}$ denotes the Pauli matrices. The generators are normalized such that $\operatorname{tr}\left(t_{a} t_{b}\right)=\delta_{a b} / 2, a, b=0, \ldots, 3$.

Left- and right-handed chiral transformations act linearly on the fields (1) according to

$$
\Phi \stackrel{U(2)_{L} \times U(2)_{R}}{\longrightarrow} U_{L} \Phi U_{R}^{\dagger} .
$$

The most general globally chirally symmetric Lagrangian that contains operators of dimension (up to) four and reproduces the chiral symmetry breaking pattern found in nature is given by

$$
\begin{aligned}
\mathcal{L}_{O(4)}= & \operatorname{tr}\left\{\left(\partial^{\mu} \Phi\right)^{\dagger} \partial_{\mu} \Phi\right\}-m_{0}^{2} \operatorname{tr}\left\{\Phi^{\dagger} \Phi\right\} \\
& -\lambda\left(\operatorname{tr}\left\{\Phi^{\dagger} \Phi\right\}\right)^{2}+\operatorname{tr}\left\{H\left(\Phi^{\dagger}+\Phi\right)\right\},
\end{aligned}
$$


where, assuming exact isospin symmetry, $H=h_{\mathrm{ESB}} t_{0}$ and $h_{\mathrm{ESB}} \sim m_{u}=m_{d}$. The explicit breaking of chiral symmetry due to nonvanishing quark masses is modeled by the term

$$
\operatorname{tr}\left\{H\left(\Phi^{\dagger}+\Phi\right)\right\}=h_{\mathrm{ESB}} \sigma,
$$

which tilts the potential into the $\sigma$-direction. Evaluating the traces, Eq. (3) becomes

$$
\begin{aligned}
\mathcal{L}_{O(4)}= & \frac{1}{2}\left(\partial_{\mu} \sigma\right)^{2}+\frac{1}{2}\left(\partial_{\mu} \vec{\pi}\right)^{2}-\frac{m_{0}^{2}}{2}\left(\sigma^{2}+\vec{\pi}^{2}\right) \\
& -\frac{\lambda}{4}\left(\sigma^{2}+\vec{\pi}^{2}\right)^{2}+h_{\mathrm{ESB}} \sigma .
\end{aligned}
$$

The spontaneous breaking of chiral symmetry is reflected in a nonvanishing vacuum expectation value $\sigma_{0}$ of the $\sigma$ field. The physical excitations of this field, corresponding to the $\sigma$ meson, are described by performing a shift in the Lagrangian (5),

$$
\sigma \rightarrow \sigma_{0}+\sigma
$$

From this, one obtains the tree-level masses of the different mesons from terms quadratic in the fields,

$$
\begin{gathered}
m_{\pi}^{2}=-m_{0}^{2}+\lambda \sigma_{0}^{2}, \\
m_{\sigma}^{2}=-m_{0}^{2}+3 \lambda \sigma_{0}^{2} .
\end{gathered}
$$

As presented in Ref. [27], the low-energy limit of the eLSM can be obtained by successively integrating out all fields heavier than the pion. It turned out that this calculation can be performed analytically, if one restricts oneself to tree level. In this way, the low-energy effective Lagrangian of the eLSM in the $O(4)$ limit, which assumes the same mathematical structure as ChPT, can be written as

$$
\begin{aligned}
\mathcal{L}_{O(4), \mathrm{eff}}= & \frac{1}{2}\left(\partial_{\mu} \vec{\pi}\right)^{2}-\frac{1}{2} m_{\pi}^{2} \vec{\pi}^{2}+C_{1, O(4)}\left(\vec{\pi}^{2}\right)^{2} \\
& +C_{2, O(4)}\left(\vec{\pi} \cdot \partial_{\mu} \vec{\pi}\right)^{2}+C_{3, O(4)}\left[\left(\partial_{\mu} \vec{\pi}\right) \cdot \partial^{\mu} \vec{\pi}\right]^{2} \\
& +\mathcal{O}\left(\pi^{6}, \partial^{6}\right) .
\end{aligned}
$$

Using the tree-level masses, the parameters $\lambda$ and $m_{0}$ can be eliminated from the expressions for the low-energy couplings of the $O(4)$ LSM,

$$
\begin{aligned}
C_{1, O(4)} & =\frac{\left(m_{\sigma}^{2}-m_{\pi}^{2}\right)^{2}}{8 m_{\sigma}^{2} \sigma_{0}^{2}}\left(1-\frac{m_{\sigma}^{2}}{m_{\sigma}^{2}-m_{\pi}^{2}}\right), \\
C_{2, O(4)} & =\frac{\left(m_{\sigma}^{2}-m_{\pi}^{2}\right)^{2}}{2 m_{\sigma}^{4} \sigma_{0}^{2}}, \\
C_{3, O(4)} & =\frac{\left(m_{\sigma}^{2}-m_{\pi}^{2}\right)^{2}}{2 m_{\sigma}^{6} \sigma_{0}^{2}} .
\end{aligned}
$$

These expressions slightly differ from the ones quoted in Ref. [27], where the equation of motion for the free pion field was used to derive the low-energy couplings. Note that, for further purpose, we have separated the first term in parentheses in Eq. (10), which arises from integrating out the $\sigma$ field, from the second one, which arises from the fourpion interaction in the tree-level potential.

\section{B. Functional renormalization group}

The FRG is an implementation of the Wilsonian renormalization principle. Changing from one energy scale to another, the integration of quantum and statistical fluctuations is performed momentum shell by momentum shell. The renormalization procedure thereby connects the microscopic interactions at an ultraviolet (UV) cutoff scale $\Lambda$ with the macroscopic physics through a sequence of effective theories.

Specifically, the FRG formulates a quantum field theory in terms of a differential equation. It focuses on the scale evolution of the effective average action $\Gamma_{k}$, where $k$ denotes the infrared (IR) cutoff introduced to the theory by adding a regulator function $R_{k}$ that acts as a momentumdependent mass. The $k$-dependent $\Gamma_{k}$ interpolates between the renormalized classical action $S=\Gamma_{k \rightarrow \Lambda}$ and the full quantum effective action $\Gamma=\Gamma_{k \rightarrow 0}$ in the IR limit, where all fluctuations are integrated out. The effective action $\Gamma$ is the generating functional of one-particle irreducible vertex functions, thus containing all information about the quantum theory.

For the upcoming FRG analysis, we switch to Euclidean spacetime with a finite volume $\mathcal{V}$, leading to a discrete momentum spectrum. Lorentz indices $\mu=0,1,2,3$ appear as lower indices. Furthermore, spacetime integrations are indicated by a short-hand notation,

$$
\int_{\mathcal{V}} \mathrm{d}^{4} x=\int_{x}
$$

Finally, we take the limit $\mathcal{V} \rightarrow \infty$ in all calculations.

The scale dependence of the effective average action is dictated by the Wetterich equation [28],

$$
\begin{aligned}
\partial_{k} \Gamma_{k} & =\frac{1}{2} \operatorname{tr}\left[\partial_{k} R_{k}\left(\Gamma_{k}^{(2)}+R_{k}\right)^{-1}\right] \\
& =\frac{1}{2}(,,
\end{aligned}
$$

where we used a graphical interpretation of the propagator and the regulator insertion $\partial_{k} R_{k}$,

$$
\left(\Gamma_{k}^{(2)}+R_{k}\right)^{-1}=\longleftarrow, \quad \partial_{k} R_{k}=-\bigotimes
$$


The second functional derivative of $\Gamma_{k}$ with respect to the fields, $\Gamma_{k}^{(2)}$, and the regulator $R_{k}$ are matrix-valued in field and momentum space as well as in all internal spaces, such as Dirac, color, and flavor space. The propagator appearing in Eq. (14) is fully field dependent and partially dressed; i.e., it contains all fluctuations with momenta approximately larger than the RG scale $k$. This is achieved by the regulator insertion, which is typically peaked around (squared) momenta of order $k^{2}$.

An infinite tower of coupled differential equations arises from Eq. (14): The flow of the effective average action is coupled to its second functional derivative. The corresponding equation for $\Gamma_{k}^{(2)}$, in turn, involves the third and fourth derivatives and, in general, the scale evolution of $\Gamma_{k}^{(n)}$ is influenced by derivatives up to order $n+2$. To obtain a closed set of differential equations, it is therefore necessary to truncate this system. For further details on the FRG approach, we refer to Refs. [50-57].

Based on the discussion of the $O(4)$ LSM in the previous section, we choose the following ansatz for the Euclidean effective average action:

$$
\begin{aligned}
\Gamma_{k}=\int_{x}\{ & \frac{Z_{k}}{2}\left(\partial_{\mu} \varphi\right) \cdot \partial_{\mu} \varphi+U_{k}(\rho)-h_{\mathrm{ESB}} \sigma+\frac{Y_{1, k}}{8}\left[\partial_{\mu}(\varphi \cdot \varphi)\right]^{2} \\
& +\frac{Y_{2, k}}{8} \varphi^{2}\left(\partial_{\mu} \varphi\right) \cdot \partial_{\mu} \varphi-\frac{X_{1, k}}{8}\left[\left(\partial_{\mu} \varphi\right) \cdot \partial_{\mu} \varphi\right]^{2} \\
& -\frac{X_{2, k}}{8}\left[\left(\partial_{\mu} \varphi\right) \cdot \partial_{\nu} \varphi\right]^{2}-\frac{X_{3, k}}{8} \varphi \cdot\left(\partial_{\mu} \partial_{\mu} \varphi\right)\left(\partial_{\nu} \varphi\right) \cdot \partial_{\nu} \varphi \\
& -\frac{X_{4, k}}{8} \varphi^{2}\left(\partial_{\mu} \partial_{\nu} \varphi\right) \cdot \partial_{\mu} \partial_{\nu} \varphi-\frac{X_{5, k}}{8}\left(\varphi \cdot \partial_{\mu} \partial_{\mu} \varphi\right)^{2} \\
& \left.-\frac{X_{6, k}}{8} \varphi^{2}\left(\partial_{\mu} \partial_{\mu} \varphi\right)^{2}+\bar{\psi}\left(Z_{k}^{\psi} \gamma_{\mu} \partial_{\mu}+y \Phi_{5}\right) \psi\right\},
\end{aligned}
$$

with

$$
\rho=\varphi \cdot \varphi=\sigma^{2}+\vec{\pi}^{2}, \quad \Phi_{5}=\sigma t_{0}+i \gamma_{5} \vec{\pi} \cdot \vec{t} .
$$

Here, we have introduced the vector $\varphi=(\sigma, \vec{\pi})$ as well as the scale-dependent constants $Z_{k}, Y_{1, k}, Y_{2, k}, X_{1, k}, \ldots, X_{6, k}$, and $Z_{k}^{\psi}$. The factors $Z_{k}$ and $Z_{k}^{\psi}$ describe the wave-function renormalization factors for scalars and fermions, respectively. The effective potential $U_{k}$ is a function of the $O(4)$ invariant $\rho$. The Yukawa coupling $y$ is assumed to be RG-scale independent [it was shown in Ref. [17] that this is a valid approximation in order to produce the chiral logarithms for the pion decay constant and the pion mass]. The RG-scale invariant parameter $h_{\mathrm{ESB}} \neq 0$ leads to explicit symmetry breaking due to nonzero quark masses.

By definition, the effective average action (16) is a functional of the classical fields. In a very common abuse of notation we do not introduce new symbols for the classical fields. The physical vacuum expectation value in the absence of external sources will be denoted as $\sigma_{0}$. We will use this convention throughout the rest of this work.
Within the ansatz (16) we have chosen the following basis structures to span the full space of terms of order $\mathcal{O}\left(\varphi^{4}, \partial^{2}\right)$ and $\mathcal{O}\left(\varphi^{4}, \partial^{4}\right)$, respectively:

$$
\begin{gathered}
{\left[\partial_{\mu}(\varphi \cdot \varphi)\right]^{2}, \quad \varphi^{2}\left(\partial_{\mu} \varphi\right) \cdot \partial_{\mu} \varphi,} \\
{\left[\left(\partial_{\mu} \varphi\right) \cdot \partial_{\mu} \varphi\right]^{2}, \quad\left[\left(\partial_{\mu} \varphi\right) \cdot \partial_{\nu} \varphi\right]^{2},} \\
\varphi \cdot\left(\partial_{\mu} \partial_{\mu} \varphi\right)\left(\partial_{\nu} \varphi\right) \cdot \partial_{\nu} \varphi, \quad \varphi^{2}\left(\partial_{\mu} \partial_{\nu} \varphi\right) \cdot \partial_{\mu} \partial_{\nu} \varphi, \\
\left(\varphi \cdot \partial_{\mu} \partial_{\mu} \varphi\right)^{2}, \quad \varphi^{2}\left(\partial_{\mu} \partial_{\mu} \varphi\right)^{2} .
\end{gathered}
$$

Moreover, we include quark fluctuations into the FRG flow, which are not included in the analysis in Ref. [27]. Thus we extend the $O(4)$ LSM from Sec. II A to the $O(4)$ quark-meson model. However, these fermionic fluctuations do not affect the tree-level low-energy couplings in Eqs. (10)-(12).

From the general truncation (16) we obtain the specific ansatz

$$
\begin{aligned}
& \Gamma_{k}=\int_{x}\left\{\frac{Z_{k}^{\sigma}}{2}\left(\partial_{\mu} \sigma\right) \partial_{\mu} \sigma+\frac{Z_{k}^{\pi}}{2}\left(\partial_{\mu} \vec{\pi}\right) \cdot \partial_{\mu} \vec{\pi}+U_{k}(\rho)-h_{\mathrm{ESB}} \sigma\right. \\
&+C_{2, k}\left(\vec{\pi} \cdot \partial_{\mu} \vec{\pi}\right)^{2}+Z_{2, k} \vec{\pi}^{2}\left(\partial_{\mu} \vec{\pi}\right) \cdot \partial_{\mu} \vec{\pi} \\
&-C_{3, k}\left[\left(\partial_{\mu} \vec{\pi}\right) \cdot \partial_{\mu} \vec{\pi}\right]^{2}-C_{4, k}\left[\left(\partial_{\mu} \vec{\pi}\right) \cdot \partial_{\nu} \vec{\pi}\right]^{2} \\
&-C_{5, k} \vec{\pi} \cdot\left(\partial_{\mu} \partial_{\mu} \vec{\pi}\right)\left(\partial_{\nu} \vec{\pi}\right) \cdot \partial_{\nu} \vec{\pi} \\
&-C_{6, k} \vec{\pi}^{2}\left(\partial_{\mu} \partial_{\nu} \vec{\pi}\right) \cdot \partial_{\mu} \partial_{\nu} \vec{\pi}-C_{7, k}\left(\vec{\pi} \cdot \partial_{\mu} \partial_{\mu} \vec{\pi}\right)^{2} \\
&\left.-C_{8, k} \vec{\pi}^{2}\left(\partial_{\mu} \partial_{\mu} \vec{\pi}\right)^{2}+\bar{\psi}\left(Z_{k}^{\psi} \gamma_{\mu} \partial_{\mu}+y \Phi_{5}\right) \psi\right\} .
\end{aligned}
$$

Here, we have made the assignments

$$
\begin{aligned}
& Z_{k}^{\pi}=Z_{k}+\frac{Y_{2, k}}{4} \sigma_{0}^{2}, \quad Z_{k}^{\sigma}=Z_{k}+Y_{1, k} \sigma_{0}^{2}+\frac{Y_{2, k}}{4} \sigma_{0}^{2}, \\
& C_{1, k}=-\frac{\lambda_{k}}{4}, \quad C_{2, k}=\frac{1}{2} Y_{1, k}, \quad Z_{2, k}=\frac{1}{8} Y_{2, k}, \\
& C_{3, k}=\frac{1}{8} X_{1, k}, \quad C_{4, k}=\frac{1}{8} X_{2, k}, \quad C_{5, k}=\frac{1}{8} X_{3, k}, \\
& C_{6, k}=\frac{1}{8} X_{4, k}, \quad C_{7, k}=\frac{1}{8} X_{5, k}, \quad C_{8, k}=\frac{1}{8} X_{6, k} .
\end{aligned}
$$

Note that we only extracted pure pion vertices from the higher couplings $Y_{1, k}, Y_{2, k}$, and $X_{1, k}, \ldots, X_{6, k}$. This is motivated by the fact that we want to keep track of the RG-scale evolution of exactly the same expressions that were produced in the tree-level approach, cf., Eq. (9). The terms $\sim Z_{2, k}$ and $\sim C_{4, k}, \ldots, C_{8, k}$ complete the structures from Eq. (9) to a full basis set, in accordance with Eqs. (18) and (19). This allows us to unambiguously project the momentum-dependent four-pion vertex onto these structures. All other terms proportional to $Y_{1, k}, Y_{2, k}$ or $X_{1, k}, \ldots, X_{6, k}$ (i.e., momentum-dependent sigma vertices and mixed 
sigma-pion vertices) are neglected. This corresponds to an approximation to the fully $O(4)$-symmetric ansatz (16) based on the expectation that the nontrivial RG running of the higher-derivative couplings will only set in roughly below the mass threshold of the $\sigma$ field, i.e., that the latter will not significantly influence the flow in the IR. Furthermore, the respective wave-function renormalization factors for the $\sigma$ and the $\pi$ fields within this approximation, cf., the first line in Eq. (21), will split as soon as $\sigma_{0}$ assumes a nonzero value. The momentum-independent four-pion interaction $C_{1, k}\left(\vec{\pi}^{2}\right)^{2}$ is part of $U_{k}$ and, thus, not explicitly shown. We identify $C_{1, k}$ with a RG-scale-dependent version of the quartic coupling $\lambda$, cf., Eq. (21).

Equation (16) corresponds to a derivative expansion. Setting the factors $Z_{k}$ and $Z_{k}^{\psi}$ to one as well as $Y_{1, k}, Y_{2, k}$, and $X_{1, k}, \ldots, X_{6, k}$ to zero, we obtain its leading order, the LPA. In this case, the effective potential $U_{k}$ is the only $k$-dependent term. Going one step further, by taking the running of the wave-function renormalization factors $Z_{k}$ and $Z_{k}^{\psi}$ into account, the truncation is called LPA'. A general field and/or momentum dependence of $Z_{k}$ and $Z_{k}^{\psi}$ is suppressed. Hence, Eq. (20) can be understood as a combination of the LPA' with the higher couplings $C_{2, k}, Z_{2, k}$, and $C_{3, k}, \ldots, C_{8, k}$.

We define the renormalized fields and couplings as

$$
\begin{array}{rlrlrl}
\tilde{\sigma} & =\sqrt{Z_{k}^{\sigma} \sigma}, & \tilde{\vec{\pi}} & =\sqrt{Z_{k}^{\pi}} \vec{\pi}, & & \\
\tilde{\psi} & =\sqrt{Z_{k}^{\psi} \psi}, & \tilde{\bar{\psi}} & =\sqrt{Z_{k}^{\psi}} \bar{\psi}, & \tilde{h}_{\mathrm{ESB}}=\frac{h_{\mathrm{ESB}}}{\sqrt{Z_{k}^{\sigma}}}, \\
\tilde{C}_{1, k} & =\frac{C_{1, k}}{\left(Z_{k}^{\pi}\right)^{2}}, & \tilde{C}_{2, k} & =\frac{C_{2, k}}{\left(Z_{k}^{\pi}\right)^{2}}, & & \tilde{Z}_{2, k}=\frac{Z_{2, k}}{\left(Z_{k}^{\pi}\right)^{2}}, \\
\tilde{C}_{3, k} & =\frac{C_{3, k}}{\left(Z_{k}^{\pi}\right)^{2}}, & \tilde{C}_{4, k} & =\frac{C_{4, k}}{\left(Z_{k}^{\pi}\right)^{2}}, & & \tilde{C}_{5, k}=\frac{C_{5, k}}{\left(Z_{k}^{\pi}\right)^{2}}, \\
\tilde{C}_{6, k} & =\frac{C_{6, k}}{\left(Z_{k}^{\pi}\right)^{2}}, & \tilde{C}_{7, k} & =\frac{C_{7, k}}{\left(Z_{k}^{\pi}\right)^{2}}, & & \tilde{C}_{8, k}=\frac{C_{8, k}}{\left(Z_{k}^{\pi}\right)^{2}}, \\
\tilde{y}^{\sigma} & =\frac{y}{Z_{k}^{\psi} \sqrt{Z_{k}^{\sigma}}}, & \tilde{y}^{\pi} & =\frac{y}{Z_{k}^{\psi} \sqrt{Z_{k}^{\pi}}} . & &
\end{array}
$$

With these definitions, Eq. (20) can be written as

$$
\begin{aligned}
\Gamma_{k}=\int_{x}\{ & \frac{1}{2}\left(\partial_{\mu} \tilde{\sigma}\right) \partial_{\mu} \tilde{\sigma}+\frac{1}{2}\left(\partial_{\mu} \tilde{\vec{\pi}}\right) \cdot \partial_{\mu} \tilde{\vec{\pi}}+\tilde{U}_{k}-\tilde{h}_{\mathrm{ESB}} \tilde{\sigma} \\
& +\tilde{C}_{2, k}\left(\tilde{\vec{\pi}} \cdot \partial_{\mu} \tilde{\vec{\pi}}\right)^{2}+\tilde{Z}_{2, k} \tilde{\vec{\pi}}^{2}\left(\partial_{\mu} \tilde{\vec{\pi}}\right) \cdot \partial_{\mu} \tilde{\vec{\pi}} \\
& \quad-\tilde{C}_{3, k}\left[\left(\partial_{\mu} \tilde{\vec{\pi}}\right) \cdot \partial_{\mu} \tilde{\vec{\pi}}\right]^{2}-\tilde{C}_{4, k}\left[\left(\partial_{\mu} \tilde{\vec{\pi}}\right) \cdot \partial_{\nu} \tilde{\vec{\pi}}\right]^{2} \\
& \quad-\tilde{C}_{5, k} \tilde{\vec{\pi}} \cdot\left(\partial_{\mu} \partial_{\mu} \tilde{\vec{\pi}}\right)\left(\partial_{\nu} \tilde{\vec{\pi}}\right) \cdot \partial_{\nu} \tilde{\vec{\pi}}-\tilde{C}_{6, k} \tilde{\vec{\pi}}^{2}\left(\partial_{\mu} \partial_{\nu} \tilde{\vec{\pi}}\right) \\
& \cdot \partial_{\mu} \partial_{\nu} \tilde{\vec{\pi}}-\tilde{C}_{7, k}\left(\tilde{\vec{\pi}} \cdot \partial_{\mu} \partial_{\mu} \tilde{\vec{\pi}}\right)^{2}-\tilde{C}_{8, k} \tilde{\vec{\pi}}^{2}\left(\partial_{\mu} \partial_{\mu} \tilde{\vec{\pi}}\right)^{2} \\
& \left.+\tilde{\bar{\psi}}\left(\gamma_{\mu} \partial_{\mu}+\tilde{y}^{\sigma} \tilde{\sigma} t_{0}+\tilde{y}^{\pi} i \gamma_{5} \tilde{\vec{\pi}} \cdot \vec{t}\right) \tilde{\psi}\right\} .
\end{aligned}
$$

The fields and couplings in the effective potential also change accordingly, $U_{k} \rightarrow \tilde{U}_{k}$. In the IR limit, $k \rightarrow 0$, the fully renormalized expressions represent "measurable" quantities.

In this truncation, the partially conserved axial current (PCAC) relation is given by

$$
\mathcal{J}_{\mu, i}^{A}=\sqrt{\frac{Z_{k}^{\pi}}{Z_{k}^{\sigma}}} \tilde{\sigma}_{0} \partial_{\mu} \tilde{\pi}_{i}+\cdots=f_{\pi} \partial_{\mu} \tilde{\pi}_{i},
$$

where $\mathcal{J}_{\mu, i}^{A}$ denotes the axial-vector current and

$$
\tilde{\sigma}_{0}=\sqrt{Z_{k}^{\sigma}} \sigma_{0}
$$

denotes the renormalized vacuum expectation value. It is related to the pion decay constant via

$$
\tilde{\sigma}_{0}=f_{\pi} \sqrt{\frac{Z_{k}^{\sigma}}{Z_{k}^{\pi}}} .
$$

For a careful derivation of the PCAC relations in Eqs. (24) and (26), see Appendix A.

The flow equations for the scale evolution of the truncation (20) of the $O(4)$ quark-meson model are rather lengthy and thus deferred to Appendix B.

\section{Effective pion action}

Following the strategy of Ref. [27], we analytically integrate out the heavier $\tilde{\sigma}$ field to estimate the modified tree-level contribution to the low-energy couplings. This means that we have to reduce the effective action in Eq. (23),

$$
\Gamma_{k}=\Gamma_{k}[\tilde{\sigma}, \tilde{\vec{\pi}}, \tilde{\bar{\psi}}, \tilde{\psi}],
$$

to a theory solely consisting of pions,

$$
\Gamma_{k}=\Gamma_{k}[\tilde{\vec{\pi}}] .
$$

To this end, the quark fields are dropped in $\Gamma_{k}$ as they do not influence the tree-level low-energy couplings. Afterwards, we eliminate the $\tilde{\sigma}$ field in the IR by exploiting the quantum equation of motion,

$$
\frac{\delta \Gamma}{\delta \tilde{\sigma}}=0 .
$$

Using the minimum condition

$$
\frac{\partial \tilde{U}_{k}}{\partial \tilde{\sigma}}=\tilde{h}_{\mathrm{ESB}}
$$

the potential is assumed to take the form

$$
\begin{aligned}
\tilde{U}_{k}= & \frac{1}{2} M_{\sigma, k}^{2} \tilde{\sigma}^{2}+\frac{1}{2} M_{\pi, k}^{2} \tilde{\vec{\pi}}^{2}+\frac{\tilde{\lambda}_{1, k}}{4} \tilde{\sigma}^{4}+\tilde{\lambda}_{1, k} \tilde{\sigma}_{0} \tilde{\sigma}^{3} \\
& +\frac{\tilde{\lambda}_{2, k}}{4}\left(\tilde{\vec{\pi}}^{2}\right)^{2}+\frac{\tilde{\lambda}_{3, k}}{2} \tilde{\sigma}^{2} \tilde{\vec{\pi}}^{2}+\tilde{\lambda}_{3, k} \tilde{\sigma}_{0} \tilde{\sigma} \tilde{\vec{\pi}}^{2},
\end{aligned}
$$


with

$\tilde{\lambda}_{1, k}=\frac{\lambda_{k}}{\left(Z_{k}^{\sigma}\right)^{2}}, \quad \tilde{\lambda}_{2, k}=\frac{\lambda_{k}}{\left(Z_{k}^{\pi}\right)^{2}}, \quad \tilde{\lambda}_{3, k}=\frac{\lambda_{k}}{Z_{k}^{\sigma} Z_{k}^{\pi}}$.

This assumption is motivated by the tree-level approach presented in Ref. [27], where the authors restricted themselves to a potential of the form (5). The renormalized masses are defined as

$$
M_{\sigma, k}^{2}=\frac{m_{\sigma, k}^{2}}{Z_{k}^{\sigma}}, \quad M_{\pi, k}^{2}=\frac{m_{\pi, k}^{2}}{Z_{k}^{\pi}}, \quad M_{\psi, k}^{2}=\frac{m_{\psi, k}^{2}}{\left(Z_{k}^{\psi}\right)^{2}} .
$$

At tree level, only the last interaction term in Eq. (31) contributes to the equation of motion (29) of the $\tilde{\sigma}$ field,

$$
\left(\partial_{\mu} \partial_{\mu}-M_{\sigma, k}^{2}\right) \tilde{\sigma}=\tilde{\lambda}_{3, k} \tilde{\sigma}_{0} \tilde{\vec{\pi}}^{2}
$$

Solving this equation for $\tilde{\sigma}$, we find

$$
\tilde{\sigma}=-\frac{\tilde{\lambda}_{3, k} \tilde{\sigma}_{0}}{M_{\sigma, k}^{2}}\left[1+\frac{\partial_{\mu} \partial_{\mu}}{M_{\sigma, k}^{2}}+\frac{\left(\partial_{\mu} \partial_{\mu}\right)^{2}}{M_{\sigma, k}^{4}}+\mathcal{O}\left(\partial^{6}\right)\right] \tilde{\vec{\pi}}^{2} .
$$

Using this relation, we finally arrive at

$$
\begin{aligned}
& \Gamma_{k}=\int_{x}\left\{\frac{1}{2}\left(\partial_{\mu} \tilde{\vec{\pi}}\right) \cdot \partial_{\mu} \tilde{\vec{\pi}}+\frac{1}{2} M_{\pi, k}^{2} \tilde{\vec{\pi}}^{2}-\tilde{C}_{1, k}^{\text {total }}\left(\tilde{\vec{\pi}}^{2}\right)^{2}\right. \\
& +\tilde{C}_{2, k}^{\text {total }}\left(\tilde{\vec{\pi}} \cdot \partial_{\mu} \tilde{\vec{\pi}}\right)^{2}+\tilde{Z}_{2, k}^{\text {total }} \tilde{\vec{\pi}}^{2}\left(\partial_{\mu} \tilde{\vec{\pi}}\right) \cdot \partial_{\mu} \tilde{\vec{\pi}} \\
& -\tilde{C}_{3, k}^{\text {total }}\left[\left(\partial_{\mu} \tilde{\vec{\pi}}\right) \cdot \partial_{\mu} \tilde{\vec{\pi}}\right]^{2}-\tilde{C}_{4, k}^{\text {total }}\left[\left(\partial_{\mu} \tilde{\vec{\pi}}\right) \cdot \partial_{\nu} \tilde{\vec{\pi}}\right]^{2} \\
& -\tilde{C}_{5, k}^{\text {total }} \tilde{\vec{\pi}} \cdot\left(\partial_{\mu} \partial_{\mu} \tilde{\vec{\pi}}\right)\left(\partial_{\nu} \tilde{\vec{\pi}}\right) \cdot \partial_{\nu} \tilde{\vec{\pi}} \\
& -\tilde{C}_{6, k}^{\text {total }} \tilde{\vec{\pi}}^{2}\left(\partial_{\mu} \partial_{\nu} \tilde{\vec{\pi}}\right) \cdot \partial_{\mu} \partial_{\nu} \tilde{\vec{\pi}}-\tilde{C}_{7, k}^{\text {total }}\left(\tilde{\vec{\pi}} \cdot \partial_{\mu} \partial_{\mu} \tilde{\vec{\pi}}\right)^{2} \\
& \left.-\tilde{C}_{8, k}^{\text {total }} \tilde{\vec{\pi}}^{2}\left(\partial_{\mu} \partial_{\mu} \tilde{\vec{\pi}}\right)^{2}\right\} \text {. }
\end{aligned}
$$

The low-energy couplings $\tilde{C}_{i, k}^{\text {total }}$ with $i \in\{1, \ldots, 8\}$ and $\tilde{Z}_{2, k}^{\text {total }}$ are given by

$$
\begin{aligned}
& \tilde{C}_{i, k}^{\text {total }}=\tilde{C}_{i, k}^{\text {tree }}+\tilde{C}_{i, k}, \\
& \tilde{Z}_{2, k}^{\text {total }}=\tilde{Z}_{2, k}^{\text {tree }}+\tilde{Z}_{2, k},
\end{aligned}
$$

where

$$
\begin{aligned}
& \tilde{C}_{1, k}^{\text {tree }}=\frac{\left(Z_{k}^{\sigma} M_{\sigma, k}^{2}-Z_{k}^{\pi} M_{\pi, k}^{2}\right)^{2}}{8\left(Z_{k}^{\pi}\right)^{2} M_{\sigma, k}^{2} \tilde{\sigma}_{0}^{2}}, \\
& \tilde{C}_{2, k}^{\text {tree }}=\frac{\left(Z_{k}^{\sigma} M_{\sigma, k}^{2}-Z_{k}^{\pi} M_{\pi, k}^{2}\right)^{2}}{2\left(Z_{k}^{\pi}\right)^{2} M_{\sigma, k}^{4} \tilde{\sigma}_{0}^{2}}, \\
& \tilde{C}_{3, k}^{\text {tree }}=\frac{\left(Z_{k}^{\sigma} M_{\sigma, k}^{2}-Z_{k}^{\pi} M_{\pi, k}^{2}\right)^{2}}{2\left(Z_{k}^{\pi}\right)^{2} M_{\sigma, k}^{6} \tilde{\sigma}_{0}^{2}},
\end{aligned}
$$

$$
\begin{gathered}
\tilde{C}_{5, k}^{\mathrm{tree}}=\frac{\left(Z_{k}^{\sigma} M_{\sigma, k}^{2}-Z_{k}^{\pi} M_{\pi, k}^{2}\right)^{2}}{\left(Z_{k}^{\pi}\right)^{2} M_{\sigma, k}^{6} \tilde{\sigma}_{0}^{2}}, \\
\tilde{C}_{7, k}^{\mathrm{tree}}=\frac{\left(Z_{k}^{\sigma} M_{\sigma, k}^{2}-Z_{k}^{\pi} M_{\pi, k}^{2}\right)^{2}}{2\left(Z_{k}^{\pi}\right)^{2} M_{\sigma, k}^{6} \tilde{\sigma}_{0}^{2}}, \\
\tilde{Z}_{2, k}^{\mathrm{tree}}=\tilde{C}_{4, k}^{\mathrm{tree}}=\tilde{C}_{6, k}^{\mathrm{tree}}=\tilde{C}_{8, k}^{\mathrm{tree}}=0 .
\end{gathered}
$$

The superscript "tree" indicates that these loop-corrected contributions to the low-energy couplings, apart from those explicitly written down in the truncation (20), are generated by the elimination of the $\tilde{\sigma}$ field. As pointed out, this is done in close analogy to the tree-level calculation of Ref. [27].

Neglecting the flow of the wave-function renormalization factors and the scale dependence of the meson masses during the integration process,

$$
\begin{gathered}
Z_{k}^{\sigma}=Z_{k}^{\pi}=1, \quad \tilde{\sigma}_{0} \rightarrow \sigma_{0}, \\
M_{\sigma, k}^{2} \rightarrow m_{\sigma}^{2}, \quad M_{\pi, k}^{2} \rightarrow m_{\pi}^{2},
\end{gathered}
$$

we reproduce the tree-level low-energy couplings of the $O(4)$ LSM in Eqs. (10)-(12). Note that, since Eq. (39) was obtained by integrating out the $\tilde{\sigma}$ field at tree level, we only obtain the first term in parentheses in Eq. (10), while the second term in that equation corresponds to the fifth term on the right-hand side of Eq. (31).

Instead of using the equation of motion for the $\tilde{\sigma}$ field to derive the purely pionic theory, one could have arrived at the same result by adding all diagrams that are one-particle reducible with respect to $\tilde{\sigma}$-meson lines to the one-particle irreducible pion amplitudes. Obviously, this also applies to the previous result, Eqs. (10)-(12), as a special case.

\section{RESULTS}

The FRG flow equations for the effective potential, the wave-function renormalization factors, and the higher derivative couplings constitute a set of coupled partial differential equations. The effective potential is numerically solved using a grid in $\sigma^{2}$, whereas the other couplings are evaluated at the IR minimum $\sigma_{0, k_{\mathrm{IR}}} \equiv \sigma_{0}$.

We choose $\Lambda=500 \mathrm{MeV}$ as UV cutoff. Recent investigations $[42,43,49]$, which are based on the dynamical hadronization technique [53,58-60], indicate that the actual range of validity of NJL-like models like the quark-meson model is closer to $\Lambda=300 \mathrm{MeV}$. However, we also want to capture quark dynamics beyond the confinement scale $\Lambda_{\mathrm{QCD}} \simeq 200 \mathrm{MeV}$, which is also the reasoning behind choosing cutoff scales as large as $1 \mathrm{GeV}$; see, e.g., Refs. [34,38,39,42].

From a technical point of view, the inclusion of quarks is advantageous in the sense that, analogously to QCD, it allows us to start the FRG flow in an approximately symmetric regime. The fermionic fluctuations in Eq. (14) 
TABLE I. UV parameters $(\Lambda=500 \mathrm{MeV})$.

\begin{tabular}{lcccc}
\hline \hline Parameter & $m_{0}$ & $\lambda$ & $h_{\mathrm{ESB}}$ & $y$ \\
\hline Value & $500 \mathrm{MeV}$ & 1.5 & $2.2 \times 10^{6} \mathrm{MeV}^{3}$ & 9.0 \\
\hline \hline
\end{tabular}

will drive $\sigma_{0, k}$ to larger nonzero values, similar to the NJLmodel mechanism of chiral symmetry breaking, which captures the low-energy dynamics of QCD [42,43,49]. The effective potential is then initialized as follows:

$$
U_{\Lambda}\left(\sigma^{2}\right)=\frac{m_{0, \Lambda}^{2}}{2} \sigma^{2}+\frac{\lambda_{\Lambda}}{4} \sigma^{4}
$$

The parameters $m_{0, \Lambda}$ and $\lambda_{\Lambda}$ are tuned such that the IR curvature masses and the pion decay constant are consistent with experimental data [61]. The concrete parameters $m_{0, \Lambda}$ and $\lambda_{\Lambda}$ that we used to produce the numerical results of this section are summarized in Table I. The wave-function renormalization factors start at a value of one in the UV, while the higher couplings $C_{2, k}, Z_{2, k}$, and $C_{3, k}, \ldots, C_{8, k}$ are initialized as zero. They are only generated during the integration process. From the scale-dependent minimum $\sigma_{0, k}$ of the effective potential we obtain the squared meson and quark masses,

$$
\begin{aligned}
& m_{\sigma, k}^{2}=2 U_{k}^{\prime}\left(\sigma_{0, k}^{2}\right)+4 \sigma_{0, k}^{2} U_{k}^{\prime \prime}\left(\sigma_{0, k}^{2}\right), \\
& m_{\pi, k}^{2}=2 U_{k}^{\prime}\left(\sigma_{0, k}^{2}\right), \\
& m_{\psi, k}^{2}=\frac{y^{2}}{4} \sigma_{0, k}^{2} .
\end{aligned}
$$

Already at this stage of the analysis we are able to make a general statement about the investigated theory: In the symmetric phase, meaning $\sigma_{0, k}=0$, the masses of the $\sigma$ field and the pions are degenerate and the quark mass vanishes. In the presence of explicit symmetry breaking, $h_{\mathrm{ESB}} \neq 0$, this can at best be achieved approximately and $\sigma_{0, k}$ can only serve as an approximate order parameter for possible phase transitions.

In Fig. 1 we plot the renormalized masses and the vacuum expectation value as a function of the IR scale $k$. As

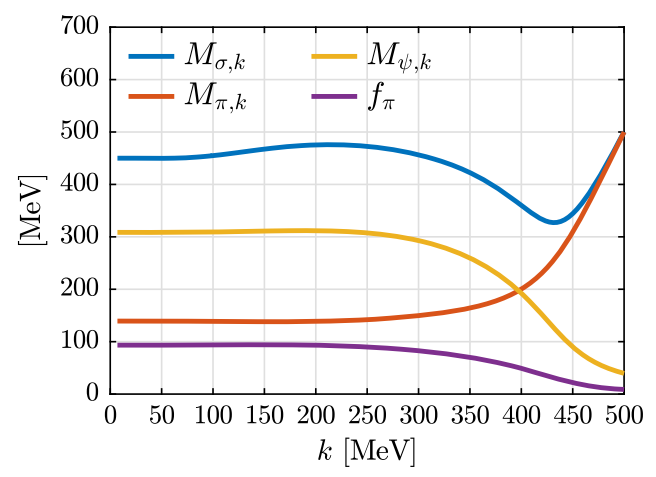

FIG. 1. Scale evolution of the renormalized meson and quark masses and the pion decay constant; $k_{\mathrm{IR}}=7 \mathrm{MeV}$. already stated in the last paragraph, $\sigma_{0, k}$ is close to zero for high energies $(k \rightarrow \Lambda)$ and, consequently, so is the quark mass $M_{\psi, k}$. In the same region, the masses of the $\sigma$ and the pions are almost identical, as expected. With a mass of around $500 \mathrm{MeV}$ they are effectively separated from the FRG flow, which is dominated by the light current quarks.

For a lower scale $k$, roughly between 400 and $450 \mathrm{MeV}$, the system undergoes a crossover transition to the spontaneously broken phase, where the minimum of the effective potential is shifted towards higher values. At a scale of $k \simeq 460 \mathrm{MeV}$, the splitting of the meson masses sets in and becomes increasingly stronger for decreasing $k$.

In the low-energy regime at $k=150 \mathrm{MeV}$ and below, the pion and the quark masses as well as $\sigma_{0, k}$ settle at their IR values. For the $\sigma$ mass, a scale dependence persists until $k<50 \mathrm{MeV}$.

We stopped the FRG flow at $k=7 \mathrm{MeV}$, an arbitrarily chosen scale close to zero (approximately 1\% of the UV cutoff) at which all $k$-dependent quantities become constant. At this point, the pions have a mass of $139.3 \mathrm{MeV}$, the $\sigma$ field has a mass of $450.1 \mathrm{MeV}$, and the quarks acquire a constituent mass of $308.5 \mathrm{MeV}$. As required by the PCAC relations (24) and (26), $\sigma_{0, k} \sqrt{Z_{k}^{\pi}}$ assumes a value of 93.4 $\mathrm{MeV}$ in the IR.

To get an impression about the correction from unrenormalized to renormalized quantities, e.g., $m_{\sigma, k} \rightarrow M_{\sigma, k}$, we show the scale dependence of $Z_{k}^{\sigma}, Z_{k}^{\pi}$, and $Z_{k}^{\psi}$ in Fig. 2 . Apparently, the overall change of $Z_{k}^{\sigma}$ and $Z_{k}^{\pi}$ is larger compared to the one of $Z_{k}^{\psi}$. The former two already split at small $\sigma_{0, k}$ according to the evaluation of their flow equations at the IR minimum. The IR values of $Z_{k}^{\sigma}$ and $Z_{k}^{\pi}$ are 1.34 and 1.47 , respectively, while that of $Z_{k}^{\psi}$ is 1.12 . This means that the meson masses are renormalized by factors of $1 / \sqrt{1.34} \simeq 0.75$ and $1 / \sqrt{1.47} \simeq 0.68$, respectively, while the quark mass is renormalized by a factor of $1 / 1.12 \simeq 0.89$. Multiplying $\sigma_{0, k}=76.9 \mathrm{MeV}$ with the factor $\sqrt{Z_{k}^{\pi}}$ yields the desired value of $f_{\pi}$, as mentioned above.

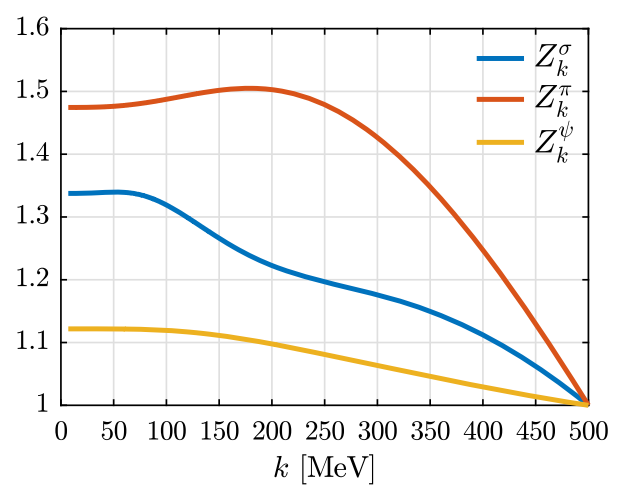

FIG. 2. Scale evolution of the wave-function renormalization factors $Z_{k}^{\sigma}, Z_{k}^{\pi}$, and $Z_{k}^{\psi} ; k_{\mathrm{IR}}=7 \mathrm{MeV}$. 


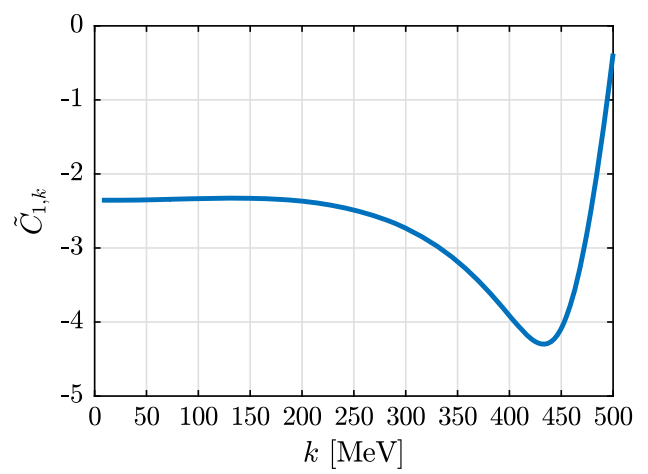

FIG. 3. Scale evolution of the renormalized coupling $\tilde{C}_{1, k}$; $k_{\mathrm{IR}}=7 \mathrm{MeV}$.

Figure 3 shows the scale dependence of the momentumindependent four-pion coupling $\tilde{C}_{1, k}$. It is initialized with a value of $-\lambda_{\Lambda} / 4=-0.3750$ at the UV cutoff (cf., Table I) and flows to its IR value of -2.3550 .

Figure 4 presents the scale evolution of the derivative couplings $\tilde{C}_{2, k}$ and $\tilde{Z}_{2, k}$. Starting from an initial value of zero in the UV, these couplings are highly sensitive to changes of the renormalization scale $k$ in the region between 450 and $500 \mathrm{MeV}$. The respective curves are especially steep for $k \rightarrow \Lambda$. This is a clear indication that the chosen cutoff scale is actually too low for the determination of these couplings. Unfortunately, larger cutoffs would exceed the range of validity of the quark-meson model as a low-energy effective theory. The fixed point-like behavior of $\tilde{C}_{2, k}$ and $\tilde{Z}_{2, k}$ below $k=250 \mathrm{MeV}$, however, can be seen as an indication for a rather mild dependence of the IR values on their initial UV values. Nevertheless, a direct calculation from QCD along the lines of Refs. [42,43,49] would be preferable for their determination.

In contrast, the evolution of $\tilde{C}_{3, k}, \ldots, \tilde{C}_{8, k}$ appears to be much flatter in the high-energy region near the UV cutoff, cf., Fig. 5. $\tilde{C}_{3, k}, \ldots, \tilde{C}_{8, k}$ substantially grow or shrink only below $250 \mathrm{MeV}$. Thus, adding to the discussion above, in this case assuming a starting value of zero at the UV scale

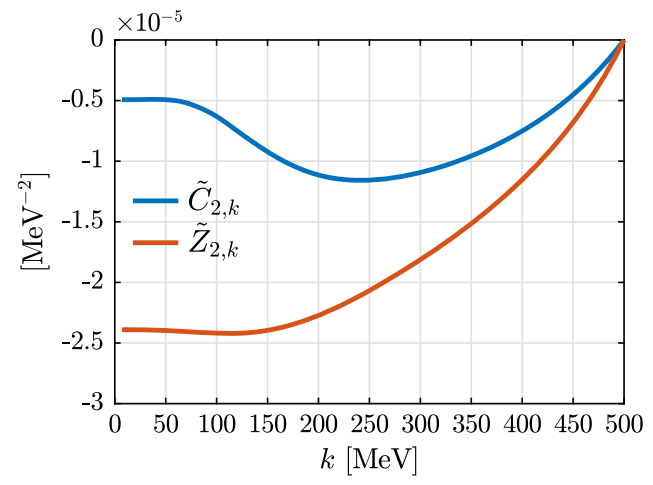

FIG. 4. Scale evolution of the renormalized couplings $\tilde{C}_{2, k}$ and $\tilde{Z}_{2, k} ; k_{\mathrm{IR}}=7 \mathrm{MeV}$.

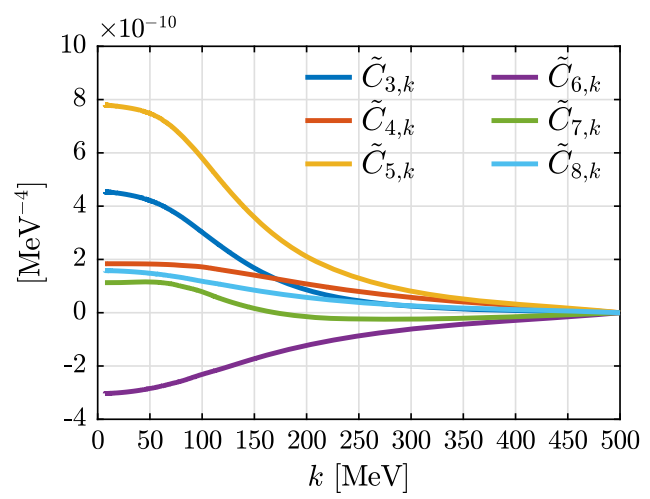

FIG. 5. Scale evolution of the renormalized couplings $\tilde{C}_{3, k}$ to $\tilde{C}_{8, k} ; k_{\mathrm{IR}}=7 \mathrm{MeV}$.

seems reasonable. Figure 5 verifies our expectation that the nontrivial running of the low-energy couplings of order $\mathcal{O}\left(\partial^{4}\right)$ with respect to the RG scale $k$ only sets in approximately below the mass threshold of the $\sigma$ field $(\simeq 400 \mathrm{MeV})$.

In the IR limit, all momentum-dependent pion selfinteractions differ from the unrenormalized couplings by a factor of $1 /\left(Z_{k}^{\pi}\right)^{2}=0.46$. The exact IR values of Figs. 3-5 are listed in the column "Trunc" in Table II. The modified tree-level contributions $\tilde{C}_{i, k}^{\text {tree }}$ with $i \in\{1, \ldots, 8\}$ and $\tilde{Z}_{2, k}^{\text {tree }}$ from Eqs. (39)-(44) are collected in the column "Tree". Their sums $\tilde{C}_{i, k}^{\text {total }}, i \in\{1, \ldots, 8\}$ and $\tilde{Z}_{2, k}^{\text {total }}$ are listed in the last column.

For the sake of providing a complete overview, Table II also contains (in column two) the numerical tree-level couplings $C_{1, O(4)}, C_{2, O(4)}$, and $C_{3, O(4)}$ from Eqs. (10)-(12), as well as $C_{5, O(4)}$ and $C_{7, O(4)}$. In contrast to Ref. [27], the latter two are also generated within the chosen basis set (18) and (19). $Z_{2}, C_{4}, C_{6}$, and $C_{8}$ vanish at tree level. All treelevel results are produced by taking the masses and the vacuum expectation value in the IR as input, i.e., $m_{\sigma}=450.1, m_{\pi}=139.3$, and $\sigma_{0}=93.4 \mathrm{MeV}$.

TABLE II. Low-energy couplings $\left(f_{\pi}=93 \mathrm{MeV}\right){ }^{\mathrm{a}}$

\begin{tabular}{lcccc}
\hline \hline & & \multicolumn{3}{c}{ FRG } \\
\cline { 3 - 5 } Coupling & Tree level & Tree & Trunc & Total \\
\hline$C_{1}$ & -0.2514 & 2.1063 & -2.3550 & -0.2487 \\
$C_{2}\left[1 / f_{\pi}^{2}\right]$ & 0.4054 & 0.3598 & -0.0426 & 0.3172 \\
$Z_{2}\left[1 / f_{\pi}^{2}\right]$ & $\ldots$ & $\ldots$ & -0.2068 & -0.2068 \\
$C_{3}\left[1 / f_{\pi}^{4}\right] \times 10^{2}$ & 1.7311 & 1.5364 & 3.3946 & 4.9310 \\
$C_{4}\left[1 / f_{\pi}^{4}\right] \times 10^{2}$ & $\ldots$ & $\ldots$ & 1.3752 & 1.3752 \\
$C_{5}\left[1 / f_{\pi}^{4}\right] \times 10^{2}$ & 3.4621 & 3.0728 & 5.8294 & 8.9023 \\
$C_{6}\left[1 / f_{\pi}^{4}\right] \times 10^{2}$ & $\ldots$ & $\ldots$ & -2.2697 & -2.2697 \\
$C_{7}\left[1 / f_{\pi}^{4}\right] \times 10^{2}$ & 1.7311 & 1.5364 & 0.8439 & 2.3804 \\
$C_{8}\left[1 / f_{\pi}^{4}\right] \times 10^{2}$ & $\ldots$ & $\ldots$ & 1.1828 & 1.1828 \\
\hline \hline
\end{tabular}

${ }^{\mathrm{a}}$ The values in the last three columns correspond to the renormalized couplings in the IR limit. 
(a)
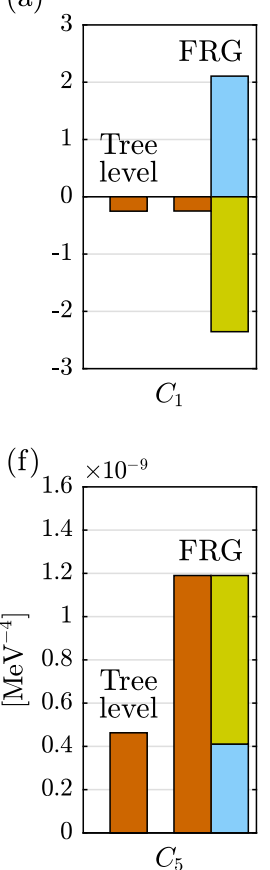

(b)

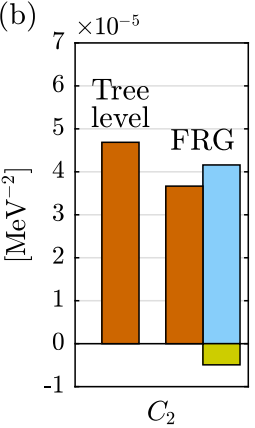

(g)

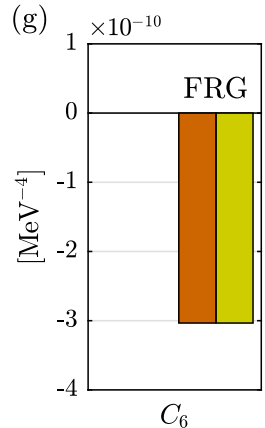

(c)

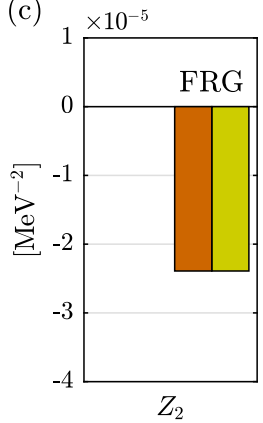

(h)

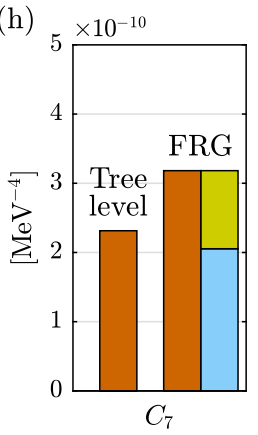

(d)
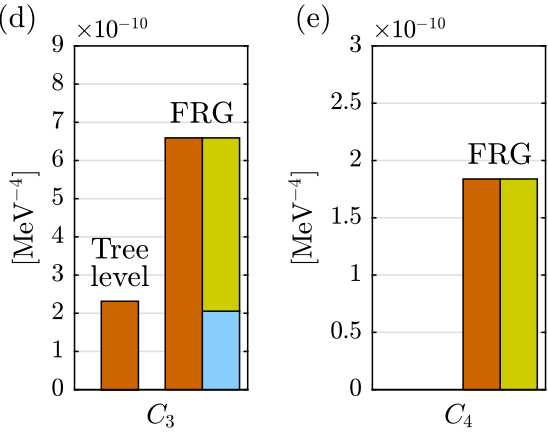

(i)

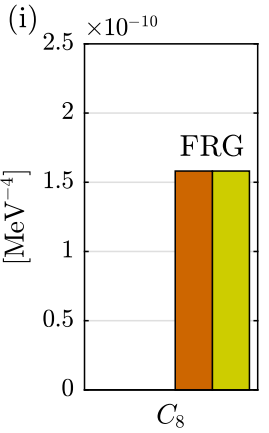

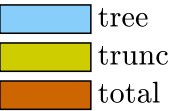

$C_{1}: \mathcal{O}\left(\partial^{0}\right)$

$C_{2}, Z_{2}: \mathcal{O}\left(\partial^{2}\right)$

$C_{3}, \ldots, C_{8}: \mathcal{O}\left(\partial^{4}\right)$

FIG. 6. Comparison of the low-energy couplings $C_{1}, \ldots, C_{8}$, and $Z_{2}$ obtained from the tree-level estimate and the FRG calculation. The contributions to the different couplings are given in separate subfigures (a)-(i). The order in the derivative expansion is summarized in the lower right corner. The bar heights correspond to the numerical values quoted in Table II. The $k$-dependent quantities are evaluated at $k_{\mathrm{IR}}=7 \mathrm{MeV}$.

The tree-level estimate for $C_{1} \simeq-0.2514$ is a good approximation. The loop-corrected coupling $\tilde{C}_{1, k}^{\text {total }}$ is only by 1.1 percent larger. The large difference between the entries in the "Tree level" and "Tree" columns for $C_{1}$ arises from the fact that the "Tree level" value contains both the contribution from integrating out the $\sigma$ field as well as that from the four-pion interaction in the potential. On the other hand, in the case of the FRG calculation the "Tree" value only contains the contribution from integrating out the $\tilde{\sigma}$ field, while the "Trunc" value arises from the potential $\tilde{U}_{k}$.

For the couplings of order $\mathcal{O}\left(\partial^{2}\right)$ and $\mathcal{O}\left(\partial^{4}\right)$, we notice that the tree-level contributions slightly decrease on account of the correction from the wave-function renormalization factors (second column compared to the third one). For $C_{2}$ we find a negative value in the column "Trunc," but all other values in the same row are positive. The total FRG results differ from the tree-level estimates by a factor of $0.78,2.85,2.57$, and 1.38 for $C_{2}, C_{3}, C_{5}$, and $C_{7}$, respectively. Hence, the corrections due to loop contributions are smallest for the momentum-independent $C_{1}$ coupling.

Figure 6 visualizes Table II in terms of a bar plot. In subfigures 6(a), 6(b), 6(d), and 6(h), both results are of the same order of magnitude $\left(\sim 10^{-1}\right.$ for $C_{1}, \sim 10^{-5} \mathrm{MeV}^{-2}$ for $C_{2}$, and $\sim 10^{-10} \mathrm{MeV}^{-4}$ for $C_{3}, C_{7}$ ), but this does not hold for $C_{5}$ in subfigure 6(f).

As the last point in this study, the amount of feedback of the scale evolution of $C_{2, k}, Z_{2, k}$, and $C_{3, k}, \ldots, C_{8, k}$ onto the masses and the wave-function renormalization is assessed by comparing the presented results to the LPA' flow. The latter is initialized with the same UV parameters as in Table I. Here, the higher-derivative couplings remain zero for all $k$. Within the LPA' truncation, the qualitative behavior of the observables in Fig. 1 is reproduced, cf., Fig. 7. The IR values (compared to the truncation beyond LPA' including the higher couplings) are found to be $M_{\sigma, k}=451.5 \quad$ (450.1), $M_{\pi, k}=136.1 \quad$ (139.3), $M_{\psi, k}=$ 309.4 (308.5), and $\sigma_{0, k} \sqrt{Z_{k}^{\pi}}=95.7$ (93.4) MeV.

At the same time, Fig. 8 reveals a significant change in the evolution of $Z_{k}^{\pi}$ within the LPA', whereas $Z_{k}^{\sigma}$ and $Z_{k}^{\psi}$ are

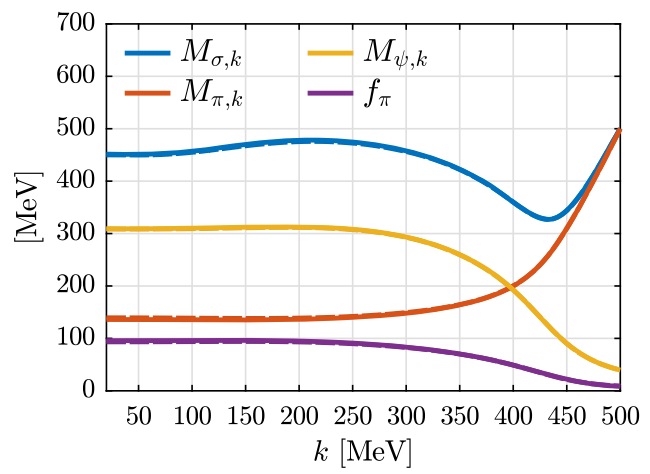

FIG. 7. Scale evolution of the renormalized meson and quark masses within the LPA' truncation. The dashed lines show the related results from Fig. $1 ; k_{\mathrm{IR}}=20 \mathrm{MeV}$. 


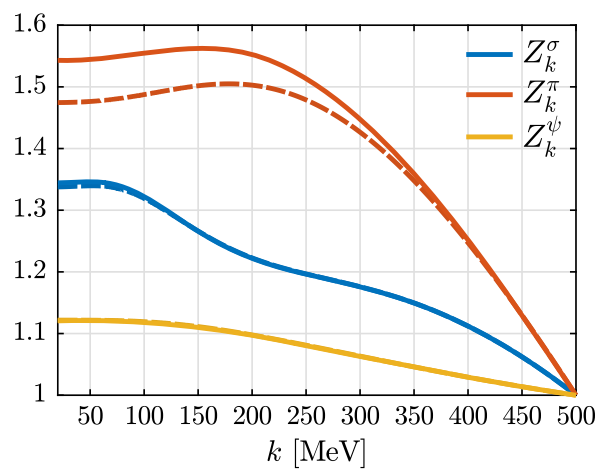

FIG. 8. Scale evolution of the wave-function renormalization factors $Z_{k}^{\sigma}, Z_{k}^{\pi}$, and $Z_{k}^{\psi}$ within the LPA' truncation. The dashed lines show the related results from Fig. $2 ; k_{\mathrm{IR}}=20 \mathrm{MeV}$.

only slightly affected: $Z_{k}^{\pi}=1.54$ (1.47), $Z_{k}^{\sigma}=1.34$ (1.34), and $Z_{k}^{\psi}=1.12(1.12)$ at $k_{\mathrm{IR}}=20 \mathrm{MeV}$. The reason for this comparatively large interference of the flow of $Z_{k}^{\pi}$ with that of $C_{2, k}, Z_{2, k}$, and $C_{3, k}, \ldots, C_{8, k}$ is immediately deduced from Eqs. (B12)-(B14) in Appendix B: The flow of $Z_{k}^{\pi}$ solely involves a four-point pion vertex, which we do not find on the right-hand sides of Eqs. (B12) and (B14). This leads to a direct coupling of the pion wave-function renormalization to the momentum-dependent pion selfinteractions. The other factors $Z_{k}^{\sigma}$ and $Z_{k}^{\psi}$ are only indirectly coupled.

\section{SUMMARY AND OUTLOOK}

In this work, we have computed the low-energy limit of the $O(4)$-symmetric quark-meson model as a limit of the eLSM. In particular, we calculated quantum corrections to the momentum-dependent four-pion interactions. As discussed in Secs. II B and III neglecting $\sigma$-dependent derivative interactions within the fully $O(4)$-symmetric model corresponds to an approximation validated by the results shown in Fig. 5.

We applied the FRG approach and compared the obtained results to a tree-level estimate. In the latter approximation, contributions to these pion self-interactions partly arise from a mapping of the low-energy effective action of the eLSM onto the one of ChPT; see Ref. [27]. We extended these contributions to a full set of basis structures for the orders $\mathcal{O}\left(\partial^{2}\right)$ and $\mathcal{O}\left(\partial^{4}\right)$.

The quantum corrections are due to the exchange of the quark as well as the meson d.o.f. of the quark-meson model as the low-energy effective theory of QCD. They have been calculated in the nonperturbative FRG approach, where the higher-derivative couplings have been treated as additional terms in a truncation beyond the well-known LPA' approximation of the quark-meson model.

We found that the overall order of magnitude of $C_{1}, C_{2}$, $C_{3}$, and $C_{7}$ is already set by their tree-level estimates, cf., Fig. 6. The loop-corrected values of $C_{1}, C_{2}$, and $C_{7}$ only differ by factors of $0.99,0.78$, and 1.38 from the tree-level estimates. However, the remaining couplings $Z_{2}, C_{4}, C_{5}$, $C_{6}$, and $C_{8}$ seem to be dominated by the loop corrections that we obtained from the FRG.

We also found a qualitative difference in the origin of the quantum contributions to $C_{2}$ and $Z_{2}$ on the one hand and $C_{3}, \ldots, C_{8}$ on the other hand. Whereas the former already receive large contributions at the initial cutoff, which are dominated by quark fluctuations, the quantum corrections to the latter are created at low RG scales, i.e., the regime that is governed by pion fluctuations. These findings encourage a study within full QCD along the lines of Refs. $[42,43,49]$ to determine the low-energy couplings, in particular $C_{2}$ and $Z_{2}$, from first-principles QCD.

On a more technical level it was demonstrated that, although the wave-function renormalization of the pion field significantly shrinks when we extend the LPA' by the higher couplings $C_{2, k}, Z_{2, k}$, and $C_{3, k}, \ldots, C_{8, k}$, the $k$-dependent meson and quark masses are rather robust against such an extension of the truncation.

It needs to be clarified how the behavior of $C_{2}$ and $Z_{2}$, as well as $C_{3}, \ldots, C_{8}$ would change if we consider the fully $O(4)$-symmetric scenario, i.e., including momentumdependent $\sigma$ self-interactions and $\sigma \pi$ couplings. Such a study is, however, beyond the scope of the present work. Moreover, in principle one has to check whether these results are reproducible under variations or extensions of the regulators (B6)-(B8), as studied in Ref. [62], and the truncation (20). Especially, the last point would be interesting in the context of the running of the low-energy couplings. In addition, it would be important to allow for a scale-dependence of the Yukawa coupling, because it is closely related to the quark mass and the pion decay constant. All of these points will be addressed in a future investigation that incorporates also effects from full QCD [49], where the dynamical hadronization technique has been applied to describe quark-antiquark bound states as mesonic d.o.f.

Finally, as repeatedly mentioned, the $O(4)$ quarkmeson model is only the specific limit of the eLSM where (axial-)vector mesons are neglected. Nevertheless, the ultimate goal of subsequent studies remains a comparison of the LECs of the full eLSM to those of ChPT. It might be therefore necessary to extend the ansatz (16) to the full effective action of the eLSM and, furthermore, to translate the presented projection of the momentumdependence of the four-pion vertex to the basis that naturally arises within the chiral expansion of ChPT.

Another important check would be to analyze the pionmass dependence of the low-energy couplings produced within the FRG, thus extending the work of Ref. [17] to higher-derivative couplings. This would constitute a nontrivial confirmation that the correct IR behavior is respected by the truncation introduced in this work, which corresponds to a partial resummation of loop diagrams. 


\section{ACKNOWLEDGMENTS}

We are very grateful to $S$. Rechenberger for helpful advice and many enlightening comments on this work. We also would like to thank M. Birse, J. Braun, D. D. Dietrich, A. Koenigstein, J. M. Pawlowski, R. D. Pisarski, F. Rennecke, B.-J. Schaefer, N. Strodthoff, and L. von Smekal for valuable discussions. J. E. acknowledges funding by the German National Academic Foundation. M. M. acknowledges funding by the Fonds zur Förderung der wissenschaftlichen Forschung Grant No. J3507-N27, the DFG Grant No. MI 2240/1-1, and the U.S. Department of Energy under Contract No. de-sc0012704. D. H. R. is partially supported by the High-end Foreign Experts project GDW20167100136 of the State Administration of Foreign Experts Affairs of China.

\section{APPENDIX A: PCAC}

In this Appendix, we present further details on the PCAC relation used in Eq. (24). First, we consider the behavior of the quark fields under infinitesimal $S U(2)_{A}$ transformations,

$$
\begin{aligned}
& \psi \rightarrow U \psi=\psi-i \alpha_{A, a} \gamma_{5} t_{a} \psi=\psi+\delta \psi, \\
& \bar{\psi} \rightarrow \bar{\psi} U=\bar{\psi}-i \bar{\psi} \alpha_{A, a} \gamma_{5} t_{a}=\bar{\psi}+\delta \bar{\psi},
\end{aligned}
$$

with $U \in S U(2)_{A}$,

$$
U=\exp \left(-i \alpha_{A, a} \gamma_{5} t_{a}\right), \quad a=1,2,3 .
$$

From the Yukawa interaction in Eq. (20), we deduce the transformation behavior of $\Phi_{5}$,

$$
\Phi_{5} \rightarrow \Phi_{5}+i \alpha_{A, a} \gamma_{5} t_{a}\left(\sigma+\sigma_{0}\right)-\alpha_{A, a} \pi_{a} t_{0} .
$$

As a consequence, we find

$$
\delta \sigma=-\alpha_{A, a} \pi_{a}, \quad \delta \pi_{a}=\alpha_{A, a}\left(\sigma+\sigma_{0}\right) .
$$

In terms of renormalized fields, these transformations read

$$
\begin{gathered}
\delta \tilde{\psi}=-i \alpha_{A, a} \gamma_{5} t_{a} \tilde{\psi}, \\
\delta \tilde{\bar{\psi}}=-i \tilde{\bar{\psi}} \alpha_{A, a} \gamma_{5} t_{a}, \\
\delta \tilde{\sigma}=-\sqrt{Z_{k}^{\sigma}} \alpha_{A, a} \frac{\tilde{\pi}_{a}}{\sqrt{Z_{k}^{\pi}}}, \\
\delta \tilde{\pi}_{a}=\sqrt{\frac{Z_{k}^{\pi}}{Z_{k}^{\sigma}}} \alpha_{A, a}\left(\tilde{\sigma}+\tilde{\sigma}_{0}\right) .
\end{gathered}
$$

The axial-vector current in Minkowski space is then given by $\left(g_{\mu \nu}\right.$ denotes the metric tensor)

$$
\begin{aligned}
& \mathcal{J}_{\mu, a}^{A}=\tilde{\tilde{\psi}} \gamma_{\mu} \gamma_{5} t_{a} \tilde{\psi}-\left(\partial_{\nu} \tilde{\sigma}\right) \sqrt{\frac{Z_{k}^{\sigma}}{Z_{k}^{\pi}}}\left(\tilde{\pi}_{a} g_{\mu}{ }^{\nu}-\frac{Z_{k}^{\pi}}{Z_{k}^{\sigma}}\left\{\tilde{C}_{5, k}\left[\left(\partial_{\rho} \tilde{\vec{\pi}}\right) \cdot \partial^{\rho} \tilde{\vec{\pi}}\right] \tilde{\pi}_{a} g_{\mu}{ }^{\nu}+2 \tilde{C}_{6, k} \tilde{\vec{\pi}}^{2} \partial_{\mu} \partial^{\nu} \tilde{\pi}_{a}+2 \tilde{C}_{7, k}\left(\tilde{\vec{\pi}} \cdot \partial_{\rho} \partial^{\rho} \tilde{\vec{\pi}}\right) \tilde{\pi}_{a} g_{\mu}{ }^{\nu}\right.\right. \\
& \left.\left.+2 \tilde{C}_{8, k} \tilde{\vec{\pi}}^{2} \partial_{\rho} \partial^{\rho} \tilde{\pi}_{a} g_{\mu}{ }^{\nu}\right\}\right)+\sqrt{\frac{Z_{k}^{\pi}}{Z_{k}^{\sigma}}}\left(\tilde{\sigma}+\tilde{\sigma}_{0}\right)\left\{\partial_{\mu} \tilde{\pi}_{a}+2 \tilde{C}_{2, k}\left(\tilde{\vec{\pi}} \cdot \partial_{\mu} \tilde{\vec{\pi}}\right) \tilde{\pi}_{a}+2 \tilde{Z}_{2, k} \tilde{\vec{\pi}}^{2} \partial_{\mu} \tilde{\pi}_{a}+\left(4 \tilde{C}_{3, k}-\tilde{C}_{5, k}\right)\left(\partial_{\nu} \tilde{\vec{\pi}}^{2} \partial_{\mu} \tilde{\pi}_{a}\right.\right. \\
& +4 \tilde{C}_{4, k}\left[\left(\partial_{\mu} \tilde{\vec{\pi}}\right) \cdot \partial_{\nu} \tilde{\vec{\pi}}\right] \partial^{\nu} \tilde{\pi}_{a}+2\left(\tilde{C}_{5, k}-\tilde{C}_{7, k}\right)\left(\tilde{\vec{\pi}} \cdot \partial_{\nu} \partial^{\nu} \tilde{\vec{\pi}}\right) \partial_{\mu} \tilde{\pi}_{a}-2 \tilde{C}_{5, k}\left[\left(\partial_{\mu} \partial_{\nu} \tilde{\vec{\pi}}\right) \cdot \partial^{\nu} \tilde{\vec{\pi}}\right] \tilde{\pi}_{a} \\
& -4 \tilde{C}_{6, k}\left(\tilde{\vec{\pi}} \cdot \partial_{\nu} \tilde{\vec{\pi}}\right) \partial_{\mu} \partial^{\nu} \tilde{\pi}_{a}-2\left(\tilde{C}_{6, k}+\tilde{C}_{8, k}\right) \tilde{\vec{\pi}}^{2} \partial_{\mu} \partial_{\nu} \partial^{\nu} \tilde{\pi}_{a}-2 \tilde{C}_{7, k}\left[\left(\partial_{\mu} \tilde{\vec{\pi}}\right) \cdot \partial_{\nu} \partial^{\nu} \tilde{\vec{\pi}}\right] \tilde{\pi}_{a}-2 \tilde{C}_{7, k}\left(\tilde{\vec{\pi}} \cdot \partial_{\mu} \partial_{\nu} \partial^{\nu} \tilde{\vec{\pi}}\right) \tilde{\pi}_{a} \\
& \left.-4 \tilde{C}_{8, k}\left(\tilde{\vec{\pi}} \cdot \partial_{\mu} \tilde{\vec{\pi}}\right) \partial_{\nu} \partial^{\nu} \tilde{\pi}_{a}\right\}
\end{aligned}
$$

\section{APPENDIX B: FLOW EQUATIONS}

We derive the flow equations for all scale-dependent parts on the right-hand side of Eq. (20) from Eq. (14) and functional derivatives thereof. To this end, we partly transform the effective average action (20) into momentum space,

$$
\begin{aligned}
& \Gamma_{k}=\mathcal{V} \sum_{q}\left[\frac{Z_{k}^{\sigma}}{2} q^{2} \sigma(-q) \sigma(q)+\frac{Z_{k}^{\pi}}{2} q^{2} \vec{\pi}(-q) \cdot \vec{\pi}(q)+\bar{\psi}(q)\left(-i Z_{k}^{\psi} \gamma_{\mu} q_{\mu}\right) \psi(q)\right]+\int_{x}\left[U_{k}(\rho)-h_{\mathrm{ESB}} \sigma\right] \\
& \quad-\mathcal{V} \sum_{q_{1}, q_{2}, q_{3}}\left\{C_{2, k} q_{1, \mu} q_{3, \mu}+Z_{2, k} q_{2, \mu} q_{3, \mu}-C_{3, k}\left(q_{1}+q_{2}+q_{3}\right)_{\mu} q_{1, \mu} q_{2, \nu} q_{3, \nu}-C_{4, k}\left(q_{1}+q_{2}+q_{3}\right)_{\mu} q_{2, \mu} q_{1, \nu} q_{3, \nu}\right. \\
&\left.-C_{5, k}\left(q_{1}+q_{2}+q_{3}\right)_{\mu} q_{1, \mu} q_{3}^{2}+C_{6, k}\left[\left(q_{1}+q_{2}+q_{3}\right)_{\mu} q_{1, \mu}\right]^{2}+C_{7, k} q_{1}^{2} q_{3}^{2}+C_{8, k}\left(q_{1}+q_{2}+q_{3}\right)^{2} q_{1}^{2}\right\} \\
& \times \pi_{i}\left(-q_{1}-q_{2}-q_{3}\right) \pi_{i}\left(q_{1}\right) \pi_{j}\left(q_{2}\right) \pi_{j}\left(q_{3}\right)+\mathcal{V} \sum_{q_{1}, q_{2}} y \bar{\psi}\left(q_{1}\right) \Phi_{5}\left(q_{1}-q_{2}\right) \psi\left(q_{2}\right) .
\end{aligned}
$$


Isospin indices $i$ and $j$ appearing twice are summed over. They replace the inner products in lines two to six of Eq. (20).

The regulators $R_{k}$ as a function of the internal momentum $q$, typically $R_{k}\left(q^{2}\right)$ for bosonic and $R_{k}(q)$ for fermionic fields, have to fulfill the requirements

$$
\begin{aligned}
& R_{k} \rightarrow 0 \text { for } k \rightarrow 0, \\
& R_{k} \rightarrow \infty \text { for } k \rightarrow \Lambda \rightarrow \infty, \\
& R_{k}>0 \text { for } q \rightarrow 0, \\
& R_{k} \rightarrow 0 \text { for } q \rightarrow \infty
\end{aligned}
$$

In this way, the high-energy modes $\left(q^{2} \gg k^{2}\right)$ are successively integrated out by lowering the scale $k$. For the soft modes $\left(q^{2} \ll k^{2}\right)$, in contrast, the regulators generate an additional mass contribution. This leads to an exclusion of these modes from the integration process.

For our analysis we take exponential-type regulators [28,62-65], namely,

$$
\begin{gathered}
R_{k}^{\sigma}\left(q^{2}\right)=Z_{k}^{\sigma} q^{2} r_{\mathrm{B}}\left(\frac{q^{2}}{k^{2}}\right), \\
R_{k}^{\pi}\left(q^{2}\right)=Z_{k}^{\pi} q^{2} r_{\mathrm{B}}\left(\frac{q^{2}}{k^{2}}\right), \\
R_{k}^{\psi}(q)=-i Z_{k}^{\psi} \gamma_{\mu} q_{\mu} r_{\mathrm{F}}\left(\frac{q^{2}}{k^{2}}\right),
\end{gathered}
$$

where the bosonic and fermionic shape functions $r_{\mathrm{B}}$ and $r_{\mathrm{F}}$ are

$$
\begin{gathered}
r_{\mathrm{B}}(x)=\frac{1}{\exp \left(x^{2}\right)-1}, \\
r_{\mathrm{F}}(x)=\sqrt{1+r_{\mathrm{B}}(x)}-1 .
\end{gathered}
$$

Acting now with the derivatives on $\Gamma_{k}$ and using the diagrammatic representations from Eq. (15), the flow equations for the effective potential $U_{k}$, the wave-function renormalizations $Z_{k}^{\sigma}, Z_{k}^{\pi}$, and $Z_{k}^{\psi}$, as well as the higher couplings $C_{2, k}, Z_{2, k}$, and $C_{3, k}, \ldots, C_{8, k}$ take the form

$$
\begin{aligned}
& \partial_{k} U_{k}=\mathcal{V}^{-1} \partial_{k} \Gamma_{k}=\mathcal{V}^{-1}\left(\begin{array}{ccc}
\frac{1}{2} & \otimes \\
\hdashline & \infty
\end{array}\right) \\
& \partial_{k} Z_{k}^{\sigma}=\left.\mathcal{V}^{-1} \frac{\mathrm{d}}{\mathrm{d} p^{2}}\right|_{p^{2}=0} \frac{\delta^{2} \partial_{k} \Gamma_{k}}{\delta \sigma(-p) \delta \sigma(p)} \\
& =\left.\mathcal{V}^{-1} \frac{\mathrm{d}}{\mathrm{d} p^{2}}\right|_{p^{2}=0}\left(\frac{1}{2}{ }^{2}\right. \\
& \partial_{k} Z_{k}^{\pi}=\left.\mathcal{V}^{-1} \frac{\mathrm{d}}{\mathrm{d} p^{2}}\right|_{p^{2}=0} \frac{\delta^{2} \partial_{k} \Gamma_{k}}{\delta \pi_{1}(-p) \delta \pi_{1}(p)} \\
& =\left.\mathcal{V}^{-1} \frac{\mathrm{d}}{\mathrm{d} p^{2}}\right|_{p^{2}=0}\left(\frac{1}{2}{ }_{\pi}\right. \\
& \underbrace{8}_{\psi} \psi(3)
\end{aligned}
$$




$$
\begin{aligned}
& \partial_{k} Z_{k}^{\psi}=\left.\frac{i}{4} \mathcal{V}^{-1} \frac{\mathrm{d}}{\mathrm{d} p^{2}}\right|_{p^{2}=0} \operatorname{tr}_{\gamma}\left[\frac{\delta}{\delta \bar{\psi}(p)} \partial_{k} \Gamma_{k} \frac{\overleftarrow{\delta}}{\delta \psi(p)} \gamma_{\mu} p_{\mu}\right]
\end{aligned}
$$

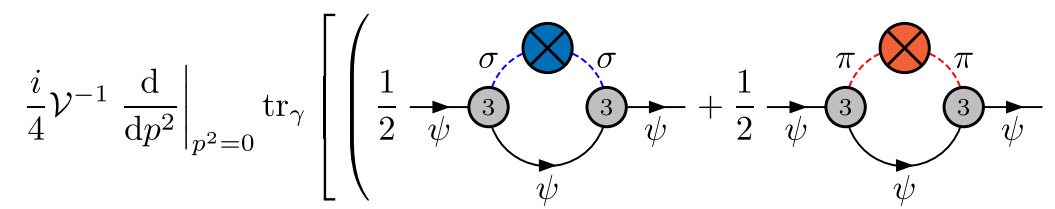

$$
\begin{aligned}
& \text { (3) (3) } \\
& \partial_{k} C_{2, k}=\left.\frac{1}{2} \mathcal{V}^{-1} \frac{\mathrm{d}}{\mathrm{d} p^{2}}\right|_{p^{2}=0} \frac{\delta^{4} \partial_{k} \Gamma_{k}}{\delta \pi_{1}(p) \delta \pi_{2}(-p) \delta \pi_{1}(0) \delta \pi_{2}(0)} \\
& =\left.\frac{1}{2} \mathcal{V}^{-1} \frac{\mathrm{d}}{\mathrm{d} p^{2}}\right|_{p^{2}=0}\left(\begin{array}{c}
2 \\
\\
\end{array}\right.
\end{aligned}
$$

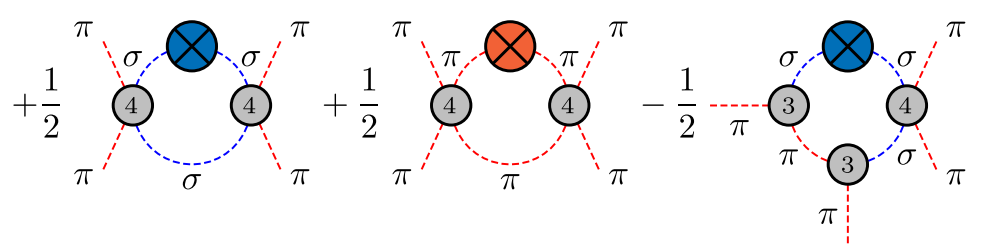

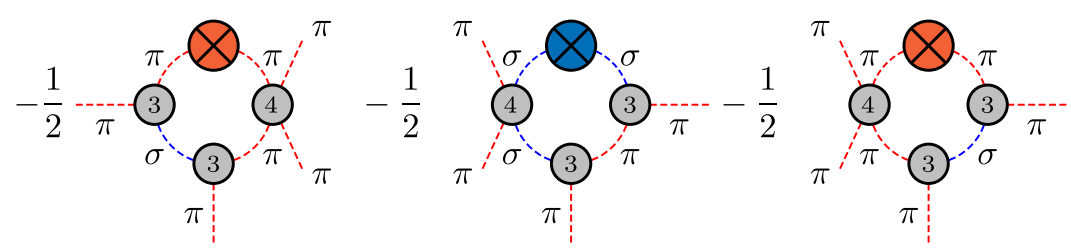

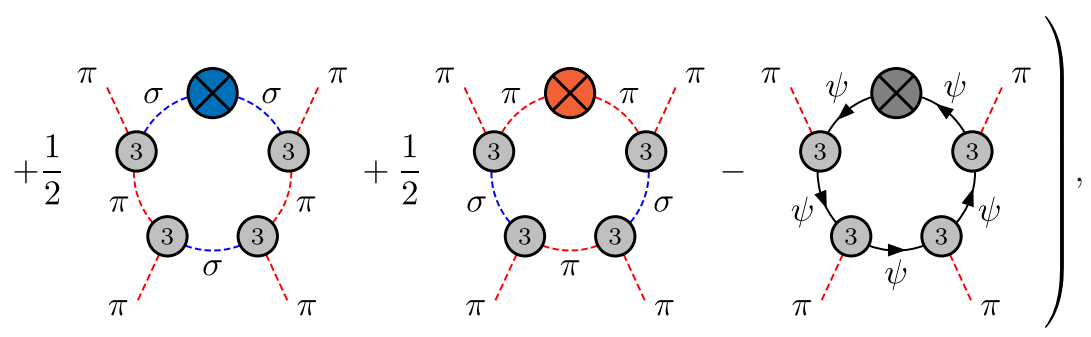

$$
\begin{aligned}
& \partial_{k} Z_{2, k}=\left.\frac{1}{4} \mathcal{V}^{-1} \frac{\mathrm{d}}{\mathrm{d} p^{2}}\right|_{p^{2}=0} \frac{\delta^{4} \partial_{k} \Gamma_{k}}{\delta \pi_{1}(p) \delta \pi_{2}(0) \delta \pi_{1}(-p) \delta \pi_{2}(0)}=\left.\frac{1}{4} \mathcal{V}^{-1} \frac{\mathrm{d}}{\mathrm{d} p^{2}}\right|_{p^{2}=0} \text { (same diagrams as in Eq. (B15)), } \\
& \partial_{k} C_{3, k}=\frac{5}{576}\left\{-\frac{208}{5} \partial_{k} C_{5, k}+112 \partial_{k} C_{6, k}-32 \partial_{k} C_{7, k}+\frac{224}{5} \partial_{k} C_{8, k}+\mathcal{V}^{-1}\left[\frac{\partial}{\partial p_{1, \mu}} \frac{\partial}{\partial p_{2, \mu}} \frac{\partial}{\partial p_{3, \nu}} \frac{\partial}{\partial p_{1, \nu}}\right.\right. \\
& \left.\left.-\frac{7}{10} \frac{\partial}{\partial p_{1, \mu}} \frac{\partial}{\partial p_{2, \mu}} \frac{\partial}{\partial p_{3, \nu}} \frac{\partial}{\partial p_{2, \nu}}\right]\left.\right|_{p_{1}=p_{2}=p_{3}=0} \frac{\delta^{4} \partial_{k} \Gamma_{k}}{\delta \pi_{1}\left(p_{1}\right) \delta \pi_{2}\left(p_{2}\right) \delta \pi_{1}\left(p_{3}\right) \delta \pi_{2}\left(-p_{1}-p_{2}-p_{3}\right)}\right\},
\end{aligned}
$$




$$
\begin{aligned}
\partial_{k} C_{4, k}=-\frac{2}{576}\{ & 16 \partial_{k} C_{5, k}+400 \partial_{k} C_{6, k}-32 \partial_{k} C_{7, k}+160 \partial_{k} C_{8, k} \\
& +\left.\mathcal{V}^{-1}\left[\frac{\partial}{\partial p_{1, \mu}} \frac{\partial}{\partial p_{2, \mu}} \frac{\partial}{\partial p_{3, \nu}} \frac{\partial}{\partial p_{1, \nu}}-\frac{5}{2} \frac{\partial}{\partial p_{1, \mu}} \frac{\partial}{\partial p_{2, \mu}} \frac{\partial}{\partial p_{3, \nu}} \frac{\partial}{\partial p_{2, \nu}}\right]\right|_{p_{1}=p_{2}=p_{3}=0} \\
& \left.\times \frac{\delta^{4} \partial_{k} \Gamma_{k}}{\delta \pi_{1}\left(p_{1}\right) \delta \pi_{2}\left(p_{2}\right) \delta \pi_{1}\left(p_{3}\right) \delta \pi_{2}\left(-p_{1}-p_{2}-p_{3}\right)}\right\}
\end{aligned}
$$$$
\partial_{k} C_{5, k}=\left.\frac{1}{96} \mathcal{V}^{-1}\left[\frac{\partial}{\partial p_{2, \mu}} \frac{\partial}{\partial p_{2, \mu}} \frac{\partial}{\partial p_{2, \nu}} \frac{\partial}{\partial p_{3, \nu}}-\frac{1}{2} \frac{\partial}{\partial p_{2, \mu}} \frac{\partial}{\partial p_{2, \mu}} \frac{\partial}{\partial p_{2, \nu}} \frac{\partial}{\partial p_{2, \nu}}\right]\right|_{p_{2}=p_{3}=0} \frac{\delta^{4} \partial_{k} \Gamma_{k}}{\delta \pi_{1}\left(-p_{2}-p_{3}\right) \delta \pi_{2}\left(p_{2}\right) \delta \pi_{1}\left(p_{3}\right) \delta \pi_{2}(0)},
$$

$$
\begin{gathered}
\partial_{k} C_{6, k}=-\frac{1}{96}\left\{-160 \partial_{k} C_{5, k}-64 \partial_{k} C_{7, k}+\left.\mathcal{V}^{-1} \frac{\partial}{\partial p_{2, \mu}} \frac{\partial}{\partial p_{4, \mu}} \frac{\partial}{\partial p_{2, \nu}} \frac{\partial}{\partial p_{4, \nu}}\right|_{p_{2}=p_{4}=0} \frac{\delta^{4} \partial_{k} \Gamma_{k}}{\delta \pi_{1}\left(-p_{2}-p_{4}\right) \delta \pi_{2}\left(p_{2}\right) \delta \pi_{1}(0) \delta \pi_{2}\left(p_{4}\right)}\right. \\
\left.-\left.\frac{1}{12} \mathcal{V}^{-1} \frac{\partial}{\partial p_{\mu}} \frac{\partial}{\partial p_{\mu}} \frac{\partial}{\partial p_{\nu}} \frac{\partial}{\partial p_{\nu}}\right|_{p=0} \frac{\delta^{4} \partial_{k} \Gamma_{k}}{\delta \pi_{1}(-p) \delta \pi_{2}(0) \delta \pi_{1}(p) \delta \pi_{2}(0)}\right\} \\
\partial_{k} C_{8, k}=\frac{1}{96}\left\{-160 \partial_{k} C_{5, k}-64 \partial_{k} C_{7, k}+\left.\left.\mathcal{V}^{-1} \frac{1}{\partial \mathcal{V}_{2, \mu}} \frac{\partial}{\partial p_{4, \mu}} \frac{\partial}{\partial p_{2, \nu}} \frac{\partial}{\partial p_{4, \nu}}\right|_{p_{2}=p_{4}=0} \frac{\partial}{\partial p_{\mu}} \frac{\partial}{\partial p_{1}\left(-p_{2}-p_{4}\right) \delta \pi_{2}\left(p_{2}\right) \delta \pi_{1}(0) \delta \pi_{2}\left(p_{4}\right)}\right|_{p=0} \frac{\delta^{4} \partial_{k} \Gamma_{k}}{\delta \pi_{1}(-p) \delta \pi_{2}(p) \delta \pi_{1}(0) \delta \pi_{2}(0)}\right. \\
\left.-\left.\frac{5}{24} \mathcal{V}^{-1} \frac{\partial}{\partial p_{\mu}} \frac{\partial}{\partial p_{\mu}} \frac{\partial}{\partial p_{\nu}} \frac{\partial}{\partial p_{\nu}}\right|_{p=0} \frac{\delta^{4} \partial_{k} \Gamma_{k}}{\delta \pi_{1}(-p) \delta \pi_{2}(0) \delta \pi_{1}(p) \delta \pi_{2}(0)}\right\}
\end{gathered}
$$

where $p$ or $p_{i}$ with $i \in\{1,2,3,4\}$ denote the external momenta and the diagrams on the right-hand side represent the sum of all possible permutations of the external legs. From Eq. (B17) on, we omitted the diagrammatic interpretation of the flow of the four-pion vertex. In the flow equations for $C_{3, k}, \ldots, C_{8, k}$, the structure of the diagrams remains unchanged, as we also indicated in Eq. (B16). Only the configuration of the external momenta is different.

The propagators of $\sigma$ and $\pi$ are highlighted in blue and red, respectively. This further applies to the regulator insertions each carrying a particular wave-function renormalization, cf., Eqs. (B6)-(B8). The black fermion lines are provided with a specific direction.

Let us remark that the four-point vertices with two external $\sigma$ legs, which one would expect in the equation for $Z_{k}^{\sigma}$, do not contribute as they are $p$ independent. The same holds true for the four-point diagram with two external pion legs and a $\sigma$ looping around. This would be part of the flow equation for $Z_{k}^{\pi}$. Five- and six-point vertices on the right of Eqs. (B15)-(B22) are omitted.

As an alternative to the differential equations for the wave-function renormalization factors we could also have presented the anomalous dimensions $\eta_{k}^{\sigma}, \eta_{k}^{\pi}$, and $\eta_{k}^{\psi}$, since

$$
\begin{gathered}
\eta_{k}^{\sigma}=-k \partial_{k} \ln Z_{k}^{\sigma}, \\
\eta_{k}^{\pi}=-k \partial_{k} \ln Z_{k}^{\pi}, \\
\eta_{k}^{\psi}=-k \partial_{k} \ln Z_{k}^{\psi} .
\end{gathered}
$$

We partly derived the flow equations by hand and additionally used the Mathematica packages FeynCalc [66,67], DoFun [68], and FormTracer [69].
[1] G. 't Hooft, Phys. Rep. 142, 357 (1986).

[2] J. S. Schwinger, Ann. Phys. (N.Y.) 2, 407 (1957).

[3] M. Gell-Mann and M. Levy, Nuovo Cimento 16, 705 (1960).
[4] S. Gasiorowicz and D. A. Geffen, Rev. Mod. Phys. 41, 531 (1969).

[5] S. Weinberg, Phys. Rev. 166, 1568 (1968). 
[6] S. R. Coleman, J. Wess, and B. Zumino, Phys. Rev. 177, 2239 (1969).

[7] C. G. Callan, Jr., S. R. Coleman, J. Wess, and B. Zumino, Phys. Rev. 177, 2247 (1969).

[8] J. Gasser and H. Leutwyler, Ann. Phys. (N.Y.) 158, 142 (1984).

[9] J. Gasser and H. Leutwyler, Nucl. Phys. B250, 465 (1985).

[10] H. Leutwyler, Ann. Phys. (N.Y.) 235, 165 (1994).

[11] V. Koch, Int. J. Mod. Phys. E 06, 203 (1997).

[12] R. D. Pisarski, arXiv:hep-ph/9503330.

[13] P. Ko and S. Rudaz, Phys. Rev. D 50, 6877 (1994).

[14] M. Urban, M. Buballa, and J. Wambach, Nucl. Phys. A697, 338 (2002).

[15] D. Bessis and J. Zinn-Justin, Phys. Rev. D 5, 1313 (1972).

[16] D. U. Jungnickel and C. Wetterich, Eur. Phys. J. C 2, 557 (1998).

[17] L. Jendges, B. Klein, H.-J. Pirner, and K. Schwenzer, arXiv: hep-ph/0608056.

[18] D. Parganlija, F. Giacosa, and D. H. Rischke, Phys. Rev. D 82, 054024 (2010).

[19] S. Janowski, D. Parganlija, F. Giacosa, and D. H. Rischke, Phys. Rev. D 84, 054007 (2011).

[20] D. Parganlija, P. Kovacs, G. Wolf, F. Giacosa, and D. H. Rischke, Phys. Rev. D 87, 014011 (2013).

[21] W. I. Eshraim, F. Giacosa, and D. H. Rischke, Eur. Phys. J. A 51, 112 (2015).

[22] S. Gallas, F. Giacosa, and D. H. Rischke, Phys. Rev. D 82, 014004 (2010).

[23] S. Gallas and F. Giacosa, Int. J. Mod. Phys. A 29, 1450098 (2014).

[24] L. Olbrich, M. Zétényi, F. Giacosa, and D. H. Rischke, Phys. Rev. D 93, 034021 (2016).

[25] S. Gallas, F. Giacosa, and G. Pagliara, Nucl. Phys. A872, 13 (2011).

[26] A. Heinz, F. Giacosa, and D. H. Rischke, Nucl. Phys. A933, 34 (2015).

[27] F. Divotgey, P. Kovacs, F. Giacosa, and D. H. Rischke, Eur. Phys. J. A 54, 5 (2018).

[28] C. Wetterich, Phys. Lett. B 301, 90 (1993).

[29] D. U. Jungnickel and C. Wetterich, Phys. Rev. D 53, 5142 (1996).

[30] D. U. Jungnickel, Quarks, Mesons and (Exact) Flow Equations, Proceedings of the International School of Physics "Enrico Fermi" Vol. 130 (IOS Press, Amsterdam, 1996).

[31] D. U. Jungnickel and C. Wetterich, arXiv:hep-ph/9610336.

[32] N. Tetradis, Nucl. Phys. A726, 93 (2003).

[33] B.-J. Schaefer and J. Wambach, Nucl. Phys. A757, 479 (2005).

[34] J. Braun, Phys. Rev. D 81, 016008 (2010).

[35] K. Kamikado, N. Strodthoff, L. von Smekal, and J. Wambach, Phys. Lett. B 718, 1044 (2013).

[36] G. Fejos, Phys. Rev. D 87, 056006 (2013).

[37] M. Grahl and D. H. Rischke, Phys. Rev. D 88, 056014 (2013).
[38] R.-A. Tripolt, N. Strodthoff, L. von Smekal, and J. Wambach, Phys. Rev. D 89, 034010 (2014).

[39] M. Mitter and B.-J. Schaefer, Phys. Rev. D 89, 054027 (2014).

[40] T. K. Herbst, M. Mitter, J. M. Pawlowski, B.-J. Schaefer, and R. Stiele, Phys. Lett. B 731, 248 (2014).

[41] M. Grahl, Phys. Rev. D 90, 117904 (2014).

[42] J. Braun, L. Fister, J. M. Pawlowski, and F. Rennecke, Phys. Rev. D 94, 034016 (2016).

[43] M. Mitter, J. M. Pawlowski, and N. Strodthoff, Phys. Rev. D 91, 054035 (2015).

[44] J. Eser, M. Grahl, and D. H. Rischke, Phys. Rev. D 92, 096008 (2015).

[45] F. Rennecke and B.-J. Schaefer, Phys. Rev. D 96, 016009 (2017).

[46] G. Almasi, R. Pisarski, and V. Skokov, Phys. Rev. D 95, 056015 (2017).

[47] C. Jung, F. Rennecke, R.-A. Tripolt, L. von Smekal, and J. Wambach, Phys. Rev. D 95, 036020 (2017).

[48] N. Strodthoff, Phys. Rev. D 95, 076002 (2017).

[49] A. K. Cyrol, M. Mitter, J. M. Pawlowski, and N. Strodthoff, Phys. Rev. D 97, 054006 (2018).

[50] T. R. Morris, Int. J. Mod. Phys. A 09, 2411 (1994).

[51] C. Bagnuls and C. Bervillier, Phys. Rep. 348, 91 (2001).

[52] J. Berges, N. Tetradis, and C. Wetterich, Phys. Rep. 363, 223 (2002).

[53] J. M. Pawlowski, Ann. Phys. (Amsterdam) 322, 2831 (2007).

[54] H. Gies, Lect. Notes Phys. 852, 287 (2012).

[55] B.-J. Schaefer and J. Wambach, Phys. Part. Nucl. 39, 1025 (2008).

[56] P. Kopietz, L. Bartosch, and F. Schütz, Lect. Notes Phys. 798, 1 (2010).

[57] L. von Smekal, Nucl. Phys. B, Proc. Suppl. 228, 179 (2012).

[58] H. Gies and C. Wetterich, Phys. Rev. D 65, 065001 (2002).

[59] H. Gies and C. Wetterich, Phys. Rev. D 69, 025001 (2004).

[60] S. Floerchinger and C. Wetterich, Phys. Lett. B 680, 371 (2009).

[61] C. Patrignani et al. (Particle Data Group), Chin. Phys. C 40, 100001 (2016).

[62] J. M. Pawlowski, M. M. Scherer, R. Schmidt, and S. J. Wetzel, Ann. Phys. (Amsterdam) 384, 165 (2017).

[63] C. Wetterich, Nucl. Phys. B352, 529 (1991).

[64] D. F. Litim, J. High Energy Phys. 11 (2001) 059.

[65] I. Nandori, J. High Energy Phys. 04 (2013) 150.

[66] R. Mertig, M. Bohm, and A. Denner, Comput. Phys. Commun. 64, 345 (1991).

[67] V. Shtabovenko, R. Mertig, and F. Orellana, Comput. Phys. Commun. 207, 432 (2016).

[68] M. Q. Huber and J. Braun, Comput. Phys. Commun. 183, 1290 (2012).

[69] A. K. Cyrol, M. Mitter, and N. Strodthoff, Comput. Phys. Commun. 219, 346 (2017). 